Florida International University FIU Digital Commons

\title{
Behavioral and Neural Mechanisms of Social Heterogeneity in Attention Deficit/Hyperactivity Disorder
}

Kailey MacNamara
kmacn001@fiu.edu

DOI: $10.25148 /$ etd.FIDC001948

Follow this and additional works at: https://digitalcommons.fiu.edu/etd

Part of the Physics Commons, and the Psychology Commons

\section{Recommended Citation}

MacNamara, Kailey, "Behavioral and Neural Mechanisms of Social Heterogeneity in Attention Deficit/Hyperactivity Disorder" (2017). FIU Electronic Theses and Dissertations. 3390.

https://digitalcommons.fiu.edu/etd/3390 


\title{
FLORIDA INTERNATIONAL UNIVERSITY
}

\author{
Miami, Florida
}

\section{BEHAVIORAL AND NEURAL MECHANISMS OF SOCIAL HETEROGENEITY IN ATTENTION DEFICIT/HYPERACTIVITY DISORDER}

\author{
A thesis in partial fulfillment of the \\ requirements for the degree of \\ MASTER OF SCIENCE \\ in \\ PHYSICS
}

by

Kailey MacNamara

2017 
To: Dean Michael R. Heithaus

College of Arts, Sciences and Education

This thesis, written by Kailey MacNamara, and entitled Behavioral and Neural Mechanisms of Social Heterogeneity in Attention Deficit/Hyperactivity Disorder, having been approved in respect to style and intellectual content, is referred to you for judgement.

We have read this dissertation and recommend that it be approved.

Erica Musser

Pete Markowitz

$\begin{array}{r}\text { Robert Laird } \\ \hline \text { Angela Laird, Major Professor }\end{array}$

Date of Defense: June 30, 2017

The thesis of Kailey MacNamara is approved.

Dean Michael R. Heithaus

College of Arts, Sciences and Education

Andrés G. Gil

Vice President for Research and Economic Development and Dean of the University Graduate School

Florida International University, 2017 
(C) Copyright 2017 by Kailey MacNamara

All rights reserved. 


\section{DEDICATION}

I dedicate this thesis to my parents and my husband. Without their love, patience, support, and understanding, the completion of this work would not have been possible. 


\section{ACKNOWLEDGMENTS}

I would like to start by thanking FIU and all of the faculty and staff who have helped me along this journey. In my eight years at FIU, I have met some incredible people who have made a lasting impression.

My first acknowledgement goes to my Quantifying Biology in the Classroom (QBIC) family (i.e., Drs. Suzanne Koptur, Bryan Dewsbury, Anya Goldina and Idaykis Rodriguez). These mentors have facilitated in my tenacious educational and personal growth (since my undergraduate studies), and are part of the reason why I love the interdisciplinary nature of science and research.

I would like to impart my deepest gratitude to my mentor Dr. Angela Laird for all of the support, wisdom, knowledge, and time that she has given to me, and for allowing my love for interdisciplinary sciences to flourish.

To my thesis committee, Drs. Robert Laird, Pete Markowitz, and Erica Musser, thank you for your patience, support, and all of your help in this process.

A special thanks to the Florida Education Fund (FEF) for honoring me as a McKnight Fellow and for helping to make graduate school less stressful and debt-free. I would also like to thank Dr. Sonja Montas-Hunter for her incessant help and words of encouragement.

To my friends and colleagues Dr. Cody Riedel, Jessica Bartley, Stephanie Rosas, and Julio Yanes for all their support, friendship, and valuable feedback. Lastly, I would like to thank my husband and family for being my constant anchor. I wouldn't be who I am today without them. 


\section{ABSTRACT OF THE THESIS \\ BEHAVIORAL AND NEURAL MECHANISMS OF SOCIAL HETEROGENEITY \\ IN ATTENTION DEFICIT/HYPERACTIVITY DISORDER}

by

Kailey MacNamara

Florida International University, 2017

Miami, Florida

Professor Angela Laird, Major Professor

Attention-deficit/hyperactivity disorder (ADHD) is one of the most common childonset neurodevelopment disorders, affecting $5 \%$ of children in the United States (American Psychiatric Association, 2013). Treatment matching in ADHD is difficult and unsatisfactory; the same general treatment algorithm is recommended for all children. It is therefore important to consider the development of specialized treatment programs based on a variety of behavioral and neurological biomarkers. Unfortunately, due to its multi-faceted classification, the heterogeneity of this behavioral disorder is under-investigated (Costa Dias et al., 2015). Scientific research in this area is especially limited as the severity of ADHD goes undiagnosed, children tend to have difficulties remaining still in MRI scanners, and the hyperactivity-impulsivity that is associated with ADHD may cause further challenges when trying to remain motionless in the scanner. Furthermore, tasks such as Facial Emotion Perception Task (FEPT) and Theory 
TABLE OF CONTENTS

CHAPTER

PAGE

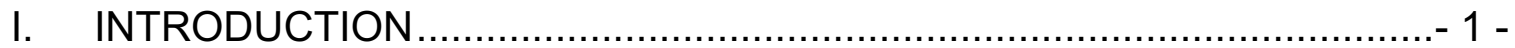

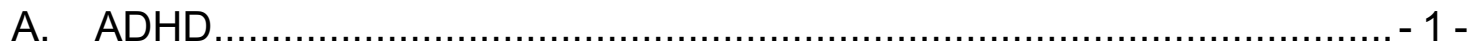

B. Function MAgnetic Resonance IMAgING ........................................ - 2 -

1. History of Magnetic Resonance Imaging ..................................... 2 -

2. Principles of Magnetic Resonance Imaging .................................. 3 -

3. Functional Magnetic Resonance Imaging Data Analysis .................. 17 -

C. COGNITIVE TASKS ....................................................................... 19 -

D. THESIS OBJECTIVES ..................................................................... 25 -

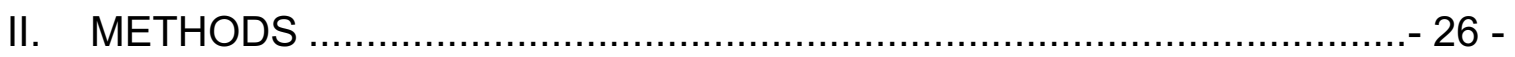

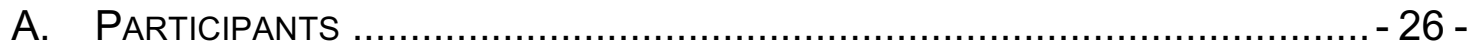

B. FMRI DATA ACQUISITION AND EXPERIMENTAL TASKS ........................... 26 -

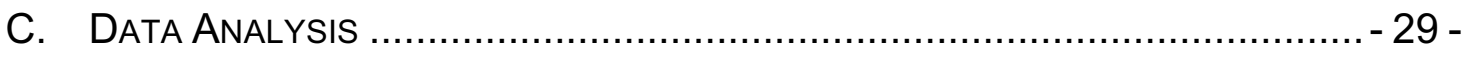

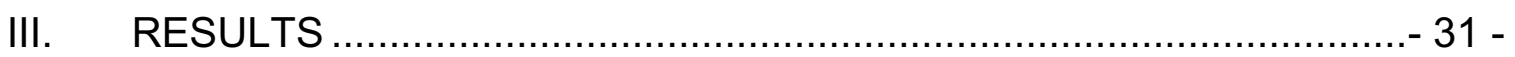

A. TASK 1 - FACIAL EMOTION PERCEPTION TASK BEHAVIORAL RESULTS ........ - 32 -

B. TASK 2 - THEORY OF MIND BEHAVIORAL RESULTS ................................ - 39 -

C. FAcIAL EMOtION PERCEPTION TASK IMAgING RESULTS .......................... 41 -

D. THEORY OF MIND TASK IMAGING RESULTS …................................... - 47 -

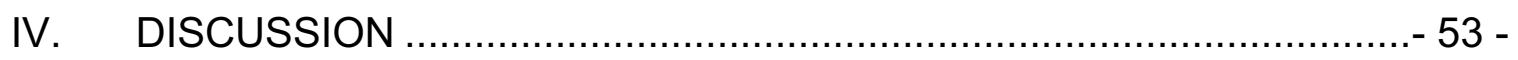

A. FACIAL EMOTION PERCEPTION TASK .............................................. - 53 -

B. THEORY OF MIND TASK …....................................................... 57 -

C. METHOdOLOGICAL CONSIDERATIONS ….............................................. 59 -

D. FUTURE WORK ….........................................................................

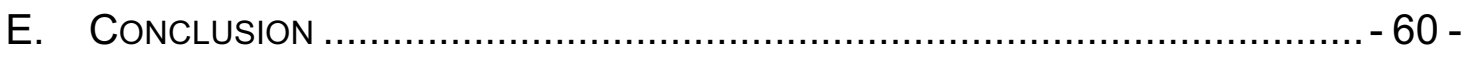

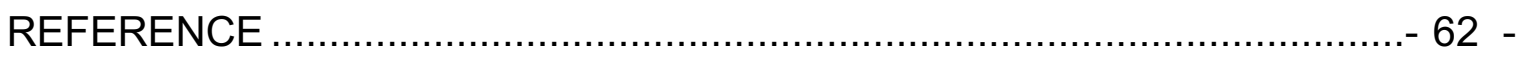




\section{LIST OF FIGURES}

FIGURE

PAGE

1. Basic Properties of Magnetic Resonance (MR) Signal Generation ............ 7

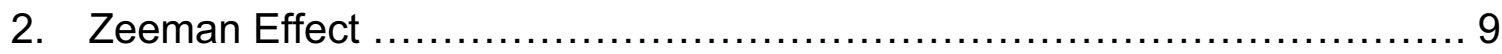

3. Facial Emotion Perception Task (FEPT) Presentation Layout ................ 21

4. Theory of Mind (ToM) Presentation Layout .................................. 23

5. Scan Protocol Layout ................................................. 30

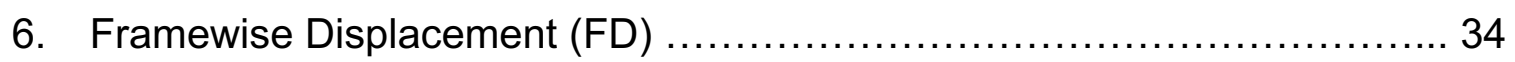

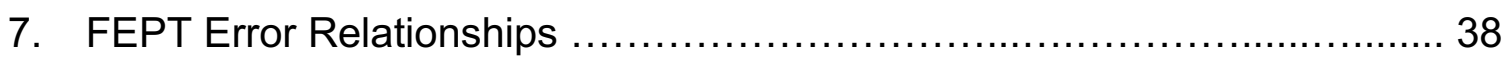

8. FEPT: Contrast Map (Emotional Faces > Rest) .............................. 43

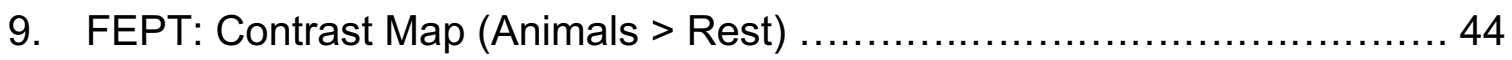

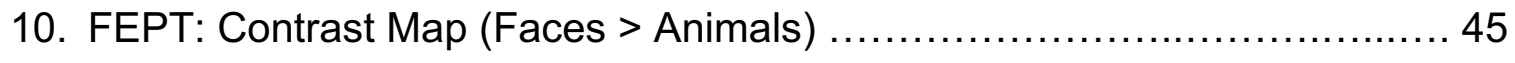

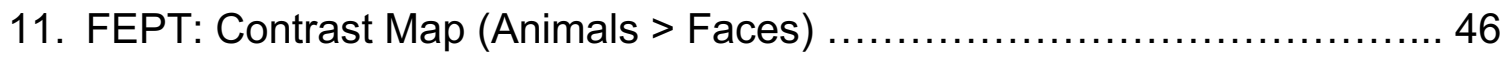

12. ToM: Contrast Map (Affective ToM (aToM) > Rest) …................... 49

13. ToM: Contrast Map (Physical Causality (PC) > Rest) .......................50

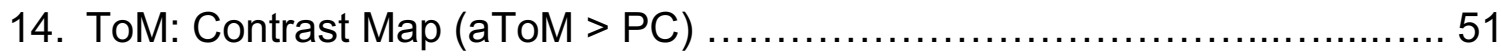

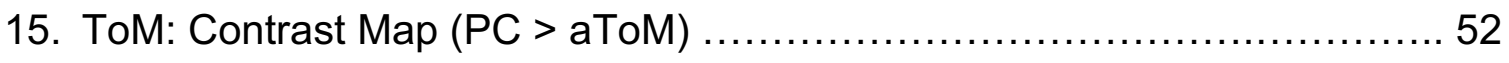




\section{INTRODUCTION}

\section{A. ADHD}

Attention-deficit/hyperactivity disorder (ADHD) is a persistent, heterogeneous neurodevelopmental disorder that affects people of all ages, and is characterized by excessive inattention and hyperactivity-impulsivity that interferes with functioning and development (American Psychiatric Association, 2013). ADHD is the most common childhood behavioral disorders (affected children - $5 \%$ of United States) and is known to be associated with cognitive deficits and social cognition impairments (e.g., perceiving emotional faces and prosody, and interpreting social cues) (American Psychiatric Association, 2013; Colby et al., 2012; Uekermann et al., 2010). Due to its recent interest (over 800 scientific papers and 3800 experiments since 1993), ADHD is being investigated for new insights using behavioral and neuroimaging modalities. Currently, the majority of functional neuroimaging studies investigating cognitive deficits associated with ADHD have focused on inhibition and working memory (Cortese et al., 2012). However, most clinical psychologists agree that ADHD is a social disorder, in which children exhibit functional impairment across multiple social domains. Unfortunately, prior research involving tasks that target these functional impairments are limited, especially for functional magnetic resonance imaging (fMRI). 


\section{B. Function Magnetic Resonance Imaging}

\section{History of Magnetic Resonance Imaging}

Nuclear magnetic resonance (NMR) was first discovered in the early 1940s by physicists Felix Bloch and Edward Purcell. Bloch and Purcell independently investigated magnetic resonance in bulk matter (i.e., looking at the absorption of energy from a time-varying magnetic field at certain frequencies) using different techniques. Purcell's lab put paraffin wax into the center of a strong, varying magnetic field and steadily increased the magnetic current until it reached its maximum, before slowly decreased it. They noticed a resonance effect from the wax in this experiment. Meanwhile Bloch's group placed water in a brass box between two poles of a strong magnet. They introduced a transmitter coil to send electromagnetic energy (i.e., radiowaves) into the sample and a detector coil to measure the water's response (i.e., emission of radio frequency). Bloch's lab also found magnetic resonance effects in their water experiment, which they called nuclear inductance. Nuclear inductance is now referred to as nuclear magnetic resonance and is modeled after Bloch's technique of a strong static magnetic field, a transmitter coil, and a detector coil. It was from this that American physicist Paul Lauterbur realized that by introducing spatial gradients (i.e., used to change the effective strength of the magnetic field in a spatial location) in the magnetic field, that images could be produced (Lauterbur, 1973). Superseding this novel approach was echo-planar imaging or EPI in 1976 by British physicist Peter Mansfield. This new approach allowed for the collection of an entire image slice at one time, instead of only looking at one-dimensional projections. Echo- 
planar imaging acquires and records an MR signal from a transmitter coil, which sends an electromagnetic pulse and then introduces time-varying magnetic field gradients. After establishing the image acquisition system, it was apparent that in order to scan humans, a scanning machine needed to be invented. That's when an American physician named Raymond Damadian, who was also experimenting with NMR, created the first human NMR scanner (in 1977). He named it the "Indomitable." This scanner, as many first generation equipment, was a step in the right direction, but needed multiple enhancements. Damadian wanted to create a strong, homogenous magnetic field that would be able to encompass a human body, but instead he was only able to produce a weak field that was only homogenous within a small dimension of volume. After two years of collecting data one voxel at a time, his group was able to create multiple images of the abdomen, upper torso, and head. This is the underpinning of imaging via MRI, and has proved to have less ionizing radiation, has provided high spatial resolution, and can obtain images in any plane through the body. These reasons are why MRI has demonstrated to be a safer, non-invasive imaging modality, when compared to other imaging modalities (i.e., x-ray, positron emission topography (PET), and computed tomography (CT)) (Huettel et al., 2004).

\section{Principles of Magnetic Resonance Imaging}

Today's magnetic resonance imaging scanners can be simplified into three main components. The first is a main static magnetic field that is produced through the bore of the scanner by a series of electromagnetic coils carrying large amounts 
of current. The last two components deal with the transmission and reception of radiofrequency (RF) via radiofrequency coils, and the image formation via alternating magnetic field strength over space (i.e., gradients). The latter step is done by turning on and off the magnetic gradient coils. In addition to these main components are the computers and hardware needed to connect to the scanner and monitor and alter the coils and therefore the magnetic field. Shimming coils are also used to compensate for inhomogeneities in the static magnetic field.

Before the magnetic resonance hardware can acquire and collect the data, it is important to understand the physical principles utilized in MR. First, we start out with the fundamental particles of the atom (i.e., electron, proton, and neutron). Together, the proton and neutron encompass the nucleus, while an electron cloud surrounds it (due to the electromagnetic attraction between a proton and electron). The proton has a positive charge and has a magnetic dipole moment. This spinning (from the proton about its axis) creates an electric current. To mathematically/physically explain this, we model this magnetic moment $(\vec{\mu})$ as a charged object (i.e., a sphere) with a spin or angular momentum. Classically, the magnetic moment is given by

$$
\vec{\mu}=I A \hat{n},
$$

where $I$ is the current, $A$ is the effective area of the "current loop," and $\hat{n}$ is the direction perpendicular to the loop. When exposed to an external magnetic field, the magnetic moment experiences a magnetic torque $(\vec{\tau})$, which aligns to the magnetic moment with the applied magnetic field (i.e., B-field; $\vec{B}$ ). The equation that quantifies this relationship is 


$$
\tau=\vec{\mu} \times \vec{B} .
$$

The rotational motion of the proton corresponds to angular momentum:

$$
\vec{J}=m \omega r^{2}
$$

where $m$ is the mass, $\omega$ is the angular velocity, and $r$ is the radius. For a quantum system, such as a proton, the magnetic moment can be described by

$$
\vec{\mu}=\gamma \vec{\jmath},
$$

where $\gamma$ is commonly referred to as the gyromagnetic ratio and can be determined to

$$
\gamma=q / 2 m
$$

and $\vec{J}$ is the angular momentum of the system. For the hydrogen nuclei (one proton), the charge is $1.60 \times 10^{-19}$ Coulombs and the mass is $1.67 \times 10^{-27}$ kilogram, which yields a gyromagnetic ratio of $4.79 \times 10^{7}$ radians/Tesla. Due to water's (and therefore, hydrogen's) abundance in the human body, hydrogen nuclei are the most common nuclei imaged in MRI.

When hydrogen, or a proton, encounters an external magnetic field $\left(\overrightarrow{B_{0}}\right)$, these protons will assume an orientation to the magnetic field: parallel state (i.e., stable equilibrium) or antiparallel state (i.e., unstable equilibrium). These two states are referred to as equilibrium states (Figure 1a). The parallel state is preferred due to the stability from the lower energy level of the spin of the proton. In either state, the proton will precess (or wobble) about the external magnetic field due to its magnetic moment and angular momentum. The frequency at which a proton precesses is known as the Larmor frequency, and is unique to each nuclei just 
like the gyromagnetic ratio. In order to calculate the Larmor frequency, we need to go back to the torque of a moving charge in an external magnetic field (i.e.,

$$
\vec{\tau}=\mu \times \overrightarrow{B_{0}} ;
$$

the rotational analogue to Newton's Second Law,

$$
\left.\sum \vec{\tau}=d \vec{J} / d t\right)
$$

We can set this equal to the change in the angular momentum over time

$$
\left(\vec{\tau}=\mu \times \overrightarrow{B_{0}}=\frac{d \vec{\jmath}}{d t}\right) .
$$

If we assume the magnetic moment is

$$
\vec{\mu}=\gamma \vec{J}
$$

we now have:

$$
\frac{d \vec{\mu}}{d t}=\gamma\left(\vec{\mu} \times \overrightarrow{B_{0}}\right)
$$

Breaking down the magnetic moment into its components we can write that the initial total magnetic moment of the spin system is:

$$
\overrightarrow{\mu(0)}=\mu_{x 0}+\mu_{y 0} \hat{y}+\mu_{z 0} \hat{z} .
$$

Transforming

$$
\frac{d \vec{\mu}}{d t}=\gamma\left(\vec{\mu} \times \overrightarrow{B_{0}}\right)
$$

into three separate scalar equations yields:

$$
\begin{aligned}
& \frac{d \mu_{x}}{d t}=\gamma \mu_{y} B_{0} ; \\
& \frac{d \mu_{y}}{d t}=\gamma \mu_{x} B_{0} ; \\
& \text { and } \frac{d \mu_{z}}{d t}=0 .
\end{aligned}
$$


a.

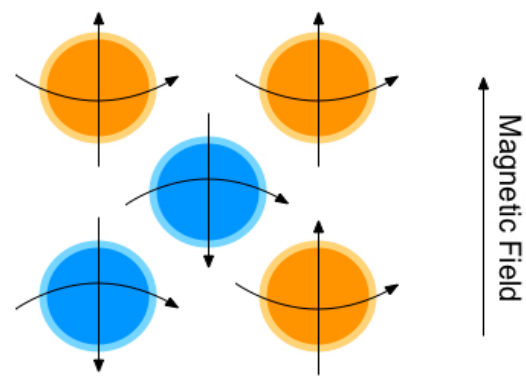

b.

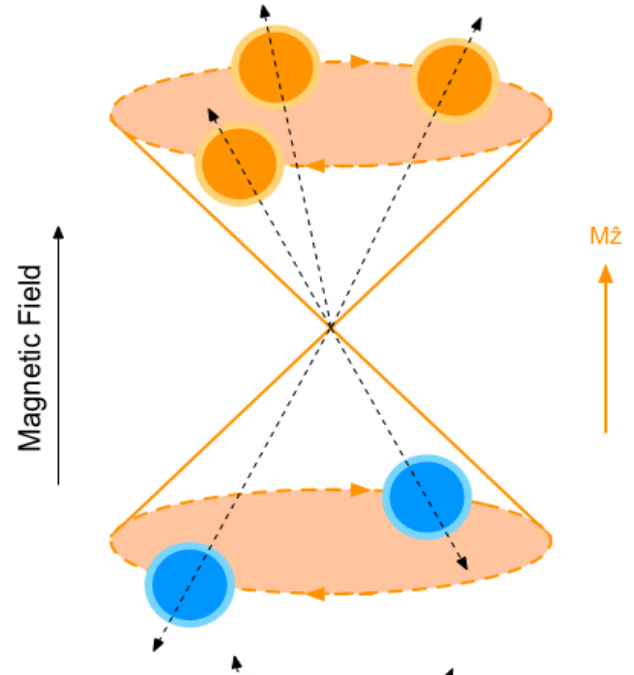

c.
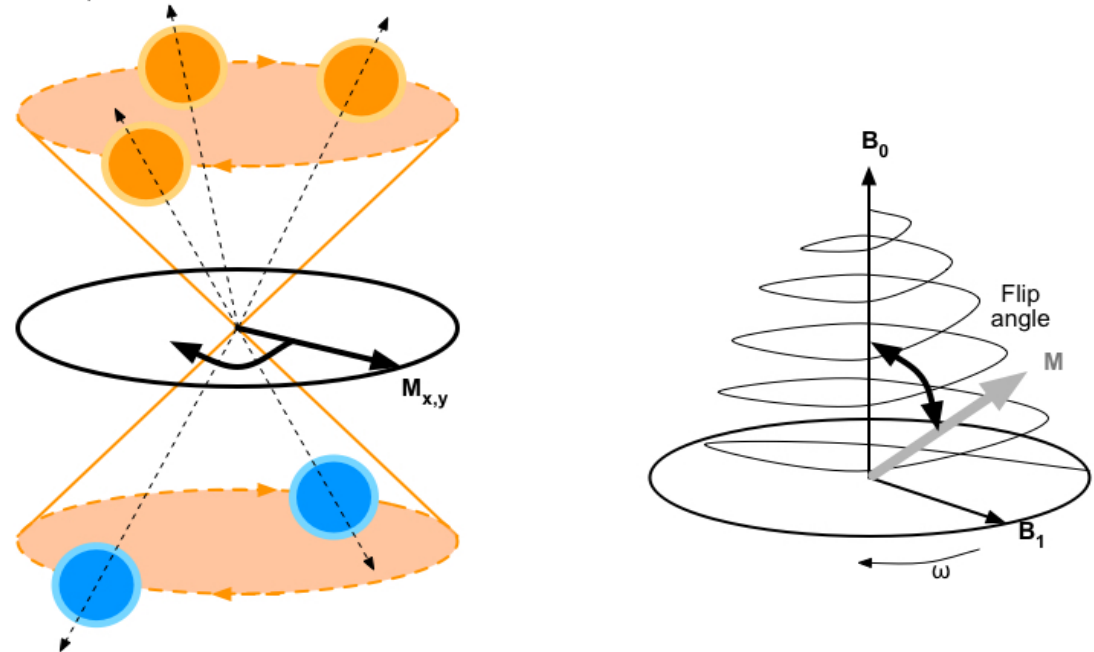

Figure 1 - Pictorial representation of some basic principles of MR signal generation adapted from Huettel et al. (2004). Part a depicts the alignment of the nuclei with nuclear magnetic resonance (NMR) properties due to the influence of external magnetic fields. The horizontal arrows represent the spins of the protons, while the vertical arrows indicate whether the proton's (in the nuclei) axis of spin align themselves along (parallel to) or against (anti-parallel to) the magnetic field. More protons tend to align their axis of spin along the magnetic field (i.e.m parallel state) and therefore cause a net magnetization that is parallel to the scanner's magnetic field (i.e., in the z-direction; part b). In part c, a radiofrequency (i.e., RF) pulse is sent out, resulting in the states to flip (i.e., parallel state flips to anti-parallel, and anti-parallel flips to parallel). This alters the net magnetization in such a way that it's orientation changes from a longitudinal (z-axis) orientation to a transverse ( $x-y$ plane) orientation. The flip angle from nutation (i.e., the wobbling path taken as the magnetization goes from longitudinal to transverse) is also shown in the second image of part c. 
After solving the set of differential equations we end up with:

$$
\overrightarrow{\mu(t)}=\left(\mu_{x 0} \cos \omega t+\mu_{y 0} \sin \omega t\right) \hat{x}+\left(\mu_{y 0} \cos \omega t-\mu_{x 0} \sin \omega t\right) \hat{y}+\mu_{z 0} \hat{z}
$$

(where the angular momentum is $\omega$ and $\gamma B_{0}$ is the Larmor frequency), implying that the magnetic moment precesses.

MR techniques are only able to measure the net magnetization $(\vec{M})$ of all the nuclei in a volume (i.e., the sum of all spins in a spin system). A net magnetization (or bulk magnetization) can be described in two components: a longitudinal component (i.e., parallel or antiparallel to the magnetic field; in the zaxis direction) and a transverse component (i.e., perpendicular to the magnetic field; in the x-y plane; Figure 1b). The transverse components cancel out, leaving the orientation of the net magnetization to solely depend on the longitudinal component (i.e., in the z-axis direction). In order to manipulate the longitudinal component, and therefore the net magnetization, the strength of the external magnetic field needs to be altered. From the Zeeman effect, it is known that when an external magnetic field is applied, the sharp spectral lines of an element (e.g., hydrogen) split into multiple closely spaced lines (Figure 2). Furthermore, with the application of a stronger external field, it requires more energy to shift from a lower-energy state to a higher-energy state, and therefore the separation of energy levels increases. The strength of the magnetic field is therefore linearly proportional to the increase net magnetization and the energy difference between the two states. It is apparent that net magnetization is a vital factor in MR signal generation, but due to the fact that net magnetization cannot be directly measured under equilibrium conditions, the system must be excited and removed 


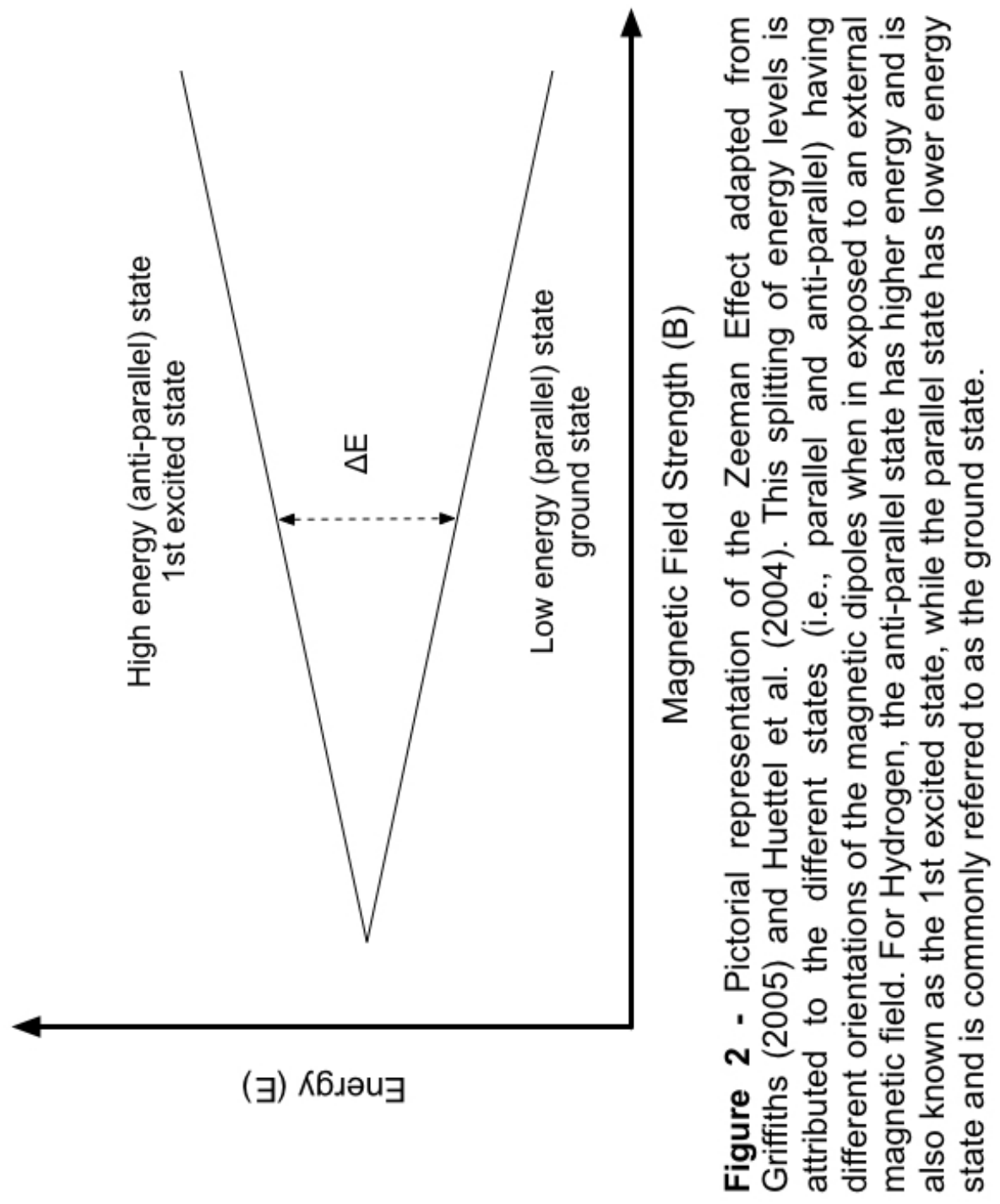


from the equilibrium state. This RF pulse excitation causes some of the spins to change from a ground (i.e., parallel) state to an excitation (i.e., anti-parallel) state (Figure 1c). In order to quantify this energy difference, the work (or energy) required to flip a spin from low-energy to high-energy

$$
\left(W=-\int_{0}^{\pi} \tau d \theta=-\int_{0}^{\pi} \mu B \sin \theta d \theta=-\left.\mu B_{0} \cos \theta\right|_{0} ^{\pi}=2 \mu B_{0}=\Delta E\right) .
$$

Comparing work to the energy difference, and using the Planck-Einstein formula, we get that the frequency of the electromagnetic pulse is equivalent to

$$
v=\frac{\Delta E}{h}=\frac{2 \mu}{h} B_{0} .
$$

Using the longitudinal component of the angular momentum of a proton

$$
\left(\frac{\hbar}{2}=\frac{h}{4 \pi}\right),
$$

the longitudinal magnetic moment is then calculated as:

$$
\mu=\gamma \frac{h}{4 \pi} \text {. }
$$

Substituting this factor, the frequency then becomes:

$$
v=\frac{2 \mu}{h} B_{0}=\frac{2}{h}\left(\gamma \frac{h}{4 \pi}\right) B_{0}=\frac{\gamma}{2 \pi} B_{0} .
$$

This frequency is equal to the Larmor frequency, and denotes that the gyromagnetic ratio of the spin and the strength of the magnetic field are the only factors that influence the frequency of the absorbed or emitted electromagnetic energy. Now that we understand the dynamics/mechanics of this nuclear spin system, it is important to discuss the net magnetization of the spin system being analyzed. First, the probability that each spin is in one of the two states is $100 \%$ (i.e.,

$$
P_{p}+P_{a}=1
$$


where $P_{p}$ is the probability of the parallel state and $P_{a}$ is the probability of being in the antiparallel state). Finding the proportion between the probability of the two spins is found to depend on the energy difference and the temperature

$$
\left(\frac{P_{p}}{P_{a}}=e^{\frac{\Delta E}{k_{b} T}}\right.
$$

where $k_{b}$ is the Boltzmann constant). Using a Taylor series approximation, we get:

$$
\frac{P_{p}}{P_{a}} \approx 1+\Delta E / k_{b} T
$$

which is known as the high-temperature approximation. Using the original probability equation

$$
\left(P_{p}+P_{a}=1\right)
$$

and the last equation, we arrive at:

$$
P_{p}-P_{a} \approx \frac{\Delta E}{2 k_{b} T}
$$

Taking this value and multiplying it by the number of protons per unit volume and the magnetic moment in the z-direction, the net magnetization is:

$$
\vec{M}=\left(\mathrm{P}_{\mathrm{p}}-\mathrm{P}_{\mathrm{a}}\right) \mathrm{n} \mu_{\mathrm{z}} \hat{z}=\frac{\Delta E}{2 k_{b} T} \mathrm{n} \mu_{\mathrm{z}} \hat{z} .
$$

When an excitation pulse is then introduced, the precession angle changes from $0^{\circ}$ and the net magnetization can be described by three scalar equations at time $\mathrm{t}=0$ :

$$
\frac{d M_{x}}{d t}=\gamma M_{y} B_{0}, \frac{d M_{y}}{d t}=-\gamma M_{x} B_{0}, \text { and } \frac{d M_{z}}{d t}=0 .
$$

After solving the set of differential equations (similar to the net magnetic moment), we get one equation that for the net magnetization at any time point: 


$$
\overrightarrow{M(t)}=M_{x 0}(\hat{x} \cos \omega t-\hat{y} \sin \omega t)+M_{y 0}(\hat{x} \sin \omega t+\hat{y} \cos \omega t)+M_{z 0} \hat{z}
$$

where $M_{x 0}, M_{y 0}$, and $M_{z 0}$ are the initial conditions. From here, it can be noticed that the net magnetization of bulk matter and the magnetic moment of a spin behave similarly. This is due to the fact that they both precess at the Larmor frequency around the main field's axis.

As mentioned before, in order to directly measure the net magnetization, an excitation is required. This excitation is delivered as a radiofrequency pulse (via a radiofrequency coil). There are two types of excitation pulses that can be administered. The first is a 90-degree excitation pulse that tips the net magnetization $90^{\circ}$ or from the longitudinal plane to the transverse plane. The second type is known as the 180-degree excitation pulse, and similar to the 90degree pulse, its name implies how large the angle of the spin tipping will be. In this type, it flips the net magnetization $180^{\circ}$ and reverses the number of highenergy and the low-energy nuclei. This 180-degree excitation pulse is not commonly used, but may sometimes be used to increasing the contrast of structural images. The generic equation to describe the excitation (or transmission) pulses is:

$$
B_{1}=B_{1} \hat{x} \cos \omega t-B_{1} \hat{y} \sin \omega t .
$$

Separating our system into two reference frames: a laboratory (or rest) frame that is aligned to the magnetic field, and a rotating frame that is rotating at Larmor frequency. In the rotating frame, the motion is represented by

$$
\widehat{x^{\prime}}=\hat{x} \cos \omega t-\hat{y} \sin \omega t \text { and } \widehat{y^{\prime}}=\hat{x} \sin \omega t+\hat{y} \cos \omega t
$$


where $x^{\prime}$ and $y^{\prime}$ represent the rotating frame and $x$ and $y$ represent the lab frame. In the rotating frame, the spins and excitation pulses become stationary. This then makes the net magnetization point along the z-axis

$$
\left(\vec{M}=M_{0} \hat{z}\right)
$$

and excitation pulse $\left(B_{1}\right)$ is now along the $x^{\prime}$-direction

$$
\left(\overrightarrow{B_{1}}=B_{1} \widehat{x^{\prime}}\right) .
$$

The combined magnetization becomes

$$
\frac{d \vec{M}}{d t}=\gamma \vec{M} \times \vec{B}
$$

(similar to what was done with the magnetic moment). The excitation pulse can either be presented at the resonant frequency (i.e., on-resonance excitation) and rotate the net magnetization from the $z$-direction towards the $x-y$ plane (transverse plane), or it can be slightly off (i.e., off-resonance excitation). A new magnetic field ( $B_{1 \text { eff }}$ ) which is affected by $B_{0}$ and $B_{1}$ is present in the spin system. This new effective excitation pulse has longitudinal $(\hat{z})$ and transverse components $\left(\widehat{x^{\prime}}\right)$ :

$$
B_{1 e f f}=\hat{z}\left(B_{0}-\frac{\omega}{\gamma}\right)+\widehat{x}^{\prime} B_{1} .
$$

When the excitation pulse is on-resonance

$$
\left(\omega=\gamma B_{0}\right),
$$

the net magnetization vector will only point in the $\widehat{x}$ direction with an angular velocity of

$$
\omega_{\text {rot }}=\gamma B_{1 e f f}=\gamma B_{1}
$$


This equation is representative of an electromagnetic field at the Larmor frequency inducing a rotation in the rotating frame. This rotation in the rotating frame is seen as the tipping (usually $90^{\circ}$ ), but in the lab frame it is seen as a spiral motion (i.e., nutation). To determine the angle to flip the net magnetization,

$$
\theta=\gamma B_{1} T
$$

is used. A larger flip angle is associated with a better image quality, but takes a longer time and has a worse signal-to-noise ratio (SNR) (i.e., the relative strength of the signal when compared to the variability in the data from sources such as fluctuations in the thermal energy in the sample and imaging hardware; Figure 1c; Huettel et al., 2004).

After the excitation pulse is applied through a radiofrequency coil, a detector coil is used to measure the de-excitation signal. The signal is measured due to the change in density of the magnetic flux $(\phi)$. From Faraday's law of induction

$$
\left(e m f=-\frac{d \phi}{d t}\right.
$$

where $\frac{d \phi}{d t}$ is the rate of change of the magnetic flux, and

$$
\phi=\int B \cdot d S
$$

is the amount of magnetic flux penetrating coil/loop), we can quantify this detection signal. This step of the receiver coil measuring the electromotive force (emf) is known as reception. This excitation-reception process is a mutual coupling that induces-receives different currents and magnetic fields as changes 
occur. The following equation describes the volume magnetic flux that is produced by the sample and detected by the receiver coil:

$$
\phi(t)=\int \overline{B_{1}} \cdot M(t) d v .
$$

From this comes the principle of reciprocity that states that the quality of an electromagnetic coil for transmission is equivalent to its quality for reception. Substituting this equation yields a new equation to calculate the electromotive force:

$$
e m f=-i \omega_{0} \int \overline{B_{1}} \cdot M(t) d v
$$

where $\omega_{0}$ (a scaling factor) came from the derivative of $M(t)$.

Following the excitation-reception process comes a relaxation. This relaxation comes about because the excitation pulses decay over time. There are two types of relaxations: longitudinal and transverse, and both cause loss of the MR signal. Longitudinal relaxation (or spin-lattice relaxation or $T_{1}$ recovery) occurs when the individual spins are losing energy (antiparallel state to parallel state) to the environment. The net magnetization returns to a direction that is parallel to the main field. This relaxation produces a smaller MR signal and a time constant $\left(T_{1}\right)$, and can be represented by:

$$
\overrightarrow{M_{z}}=\overrightarrow{M_{0}}\left(1-e^{-t / T_{1}}\right) .
$$

This $T_{1}$ recovery is used when acquiring structural (anatomical) images and is material dependent. The transverse relaxation or $T_{2}$ decay is a phenomenon that 
occurs because the transverse components of the spin lose phase coherence (i.e., they lose alignment). This intrinsic spin-spin interaction is one of two main causes for transverse relaxation and is characterized by the time constant $T_{2}$ and represented by

$$
\overrightarrow{M_{x y}}=\overrightarrow{M_{0}}\left(e^{-t / T_{2}}\right) \text {. }
$$

The second main cause for transverse relaxation arises from the inhomogeneous external magnetic field which causes differential spin effects. A loss of coherence (i.e., phase coherence- the degree of synchronicity of the nuclear spins; caused by a lack of field homogeneity) is also produced, but can be reversed by using specialized pulse sequences. When both of these effects (i.e., spin-spin interaction and field inhomogeneity) are combined together, they create a signal loss known as $T_{2}{ }^{*}$ decay, which is faster than $T_{2}$ decay. This means that the time constant $T_{2}{ }^{*}$ is smaller than $T_{2} \cdot T_{2}$ and $T_{2}{ }^{*}$ are commonly used to generate functional images due to the shorter time interval and shows greater activation (i.e., image brightness) in gray matter, which contains more cell bodies (i.e., neurons and glial cells). Combining the $T_{1}$ and $T_{2}$ processes create an equation that summarizes the MR phenomena:

$$
\frac{d \vec{M}}{d t}=\gamma \vec{M} \times \vec{B}+\frac{1}{T_{1}}\left(\overrightarrow{M_{0}}-\overrightarrow{M_{z}}\right)-\frac{1}{T_{2}}\left(\overrightarrow{M_{x}}-\overrightarrow{M_{y}}\right) .
$$

This equation is known as the Bloch equation and describes the net magnetization of a spin system in the presence of a magnetic field that varies over time (Huettel et al., 2004). 
Functional magnetic resonance imaging (fMRI) aids in exploring the neural basis of brain networks that are recruited during goal-oriented behavior by measuring changes in the local oxygenation of blood (i.e., blood oxygenation level dependent (BOLD)). This increase of local blood oxygen is caused by neurons in the brain becoming active (i.e., firing) and results in a shift in the ratio of oxygenated versus de-oxygenated hemoglobin. fMRI is able to monitor these hemodynamic responses by detecting the subtle magnetic field perturbations or changes in magnetic susceptibility due to the dynamic influx of oxygenated and de-oxygenated blood. Once processed, fMRI data may be used to identify brain regions activated during the mental task performed by a participant (Poldrack et al., 2011).

\section{Functional Magnetic Resonance Imaging Data Analysis}

The data collected from the scanner is in its rawest form and needs to be manipulated and transformed in order to be fully analyzed. The MR signal can be reconstructed into images using in-house Perl scripts that allow the raw scan data to be parsed and allocated into appropriate directories before converting the DICOM (Digital Imaging and Communications in Medicine) images into (.nii) formatted image files. Two examples of software packages commonly used to spatially normalize the brain images (i.e., transforming the data into a standard brain space): AFNI (Analysis of Functional Neurolmages) (Cox, 1996) and FSL (FMRIB Software Library; FMRIB - Functional Magnetic Resonance Imaging of the Brain) (Smith et al., 2004). For individual analyses, spatial normalization 
allows activated regions to be reported in a standard spatial coordinate system. On the group level, spatial normalization allows data across subjects to be averaged by establishing spatial correspondence between brains. There are two commonly used standardized brain templates: the Talairach atlas (Talairach and Tournoux, 1988) and the Montreal Neurological Institute (MNI) template (Collins et al., 1994). The Talairach atlas was created in 1967 by French physician Jean Talairach and his colleagues, and was based off the brain of a 60-year-old woman. The origin of the atlas is the midpoint of the anterior commissure with the $x$ - and $y$-axes defined by the horizontal plane connecting the anterior and posterior commissures (Talairach and Tournoux, 1988). The MNI template was created by the Montreal Neurological Institute and is based on structural MRIs from over one hundred subjects (Collins et al., 1994). This probabilistic template is larger than the Talairach atlas and greatest differences can be seen in the temporal lobes. The MNI152 template is a commonly used MNI template.

Once the brains are normalized, additional pre-processing operations are performed. Head motion correction is the first preprocessing step performed/completed and refers to the realignment between images of the scans across time. This step minimizes the loss of function by aligning each image in the fMRI time series to a common reference scan and then reslicing them to create realigned versions of the original data. After motion correction, non-brain removal is performed using a brain extraction (also known as skull-stripping) tool. This tool removes non-brain tissue from the image (e.g., bone, air, scalp, etc.). Spatial smoothing with a $5 \mathrm{~mm}$ full width at half maximum (FWHM) Gaussian 
kernel, high-pass temporal filtering is then used to remove artifacts associated with scanner drift (i.e., slow changes in the resonant frequency of the hydrogen atoms in the sample). This step intentionally blurs the data in order to reduce the noise, but reduces the spatial resolution in the process. Lastly, linear registration to MNI-space is performed. The first 5 volumes are then discarded to eliminate magnetization saturation effects (i.e., when an increase in the applied external magnetic field cannot increase the magnetization of the material further and the total magnetic flux density plateaus). A General Linear Modeling (GLM) analysis can then be performed to isolate the neural regions participating in each task in order to contrast different features in a task (Poldrack et al., 2011; Huettel et al., 2004).

\section{Cognitive Tasks}

While in an MRI scanner, a participant may be asked to rest for an amount of time (eyes open or closed), or they may be asked to perform a mental task. These mental tasks may require the participant to engage in motor, sensory, or cognitive behaviors. In particular, the Facial Emotion Perception Task (FEPT) (Ekman and Friesen, 1976) and Theory of Mind (ToM) (Premack and Woodruff,

1978) are cognitive tasks that require participants to identify animals, facial expressions, and correctly identify endings to stories. These cognitive tasks involve perception, judgement, and memory of social stimuli, which can then be used to further investigate potential social and behavioral deficits in children with attention deficit/hyperactivity disorder. 
The Facial Emotion Perception Task (FEPT) presents a series of alternating stacks of images with facial expressions and animals, which the participant is required to correctly identify, and is used to assess an individual's ability to categorize facial expressions (Ekman and Friesen, 1976; Rapport et al., 2002; Langenecker et al., 2007; Weisenbach et al., 2012; Figure 3). Emotion perception tasks in literature have highlighted involvement of multiple regions of neural activation in different participant groups (e.g., affected populations or typically developing controls). The task implementation (e.g., stimulus, instructions) may also vary. In one study, participants viewed two houses (similar approach as with animal pictures; both used as controls and contrasts for the emotional faces) and two emotional face on a slide (vertically or horizontally). From studying the effects of spatial attention (i.e., faces > houses) it was observed that the bilateral fusiform gyrus and the left inferior temporal gyrus were activated in healthy adults (Vuilleumier et al., 2001). Another emotion perception study required children with ADHD and typically developing children to rate the perceived threat, fear, and nose width of facial expressions (i.e., happy, angry, fearful, and neutral). This study found activations in the left amygdala when rating fear comparing children with ADHD to typically developing children (Brotman et al., 2010).

The Theory of Mind (ToM) task requires participants to select the correct ending to a cartoon story, and is used to target empathy as the participants need to attribute mental states (i.e., beliefs, intents, desires, emotions, etc.; Sebastian et al., 2012; Figure 4). In a review by Gallagher and Frith (2003), the anterior 

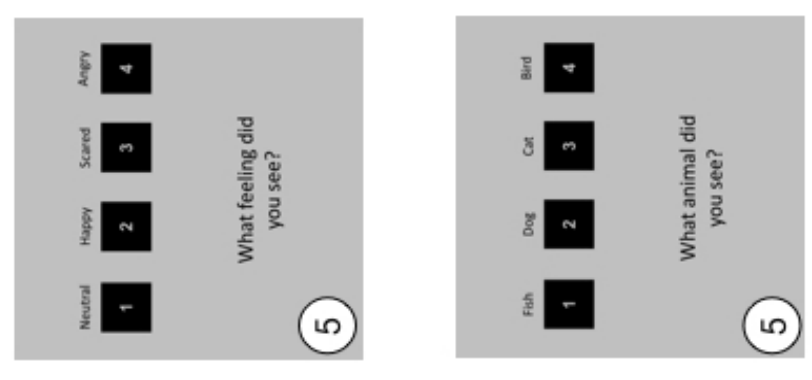

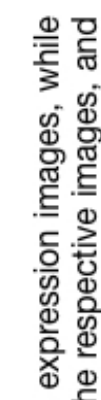
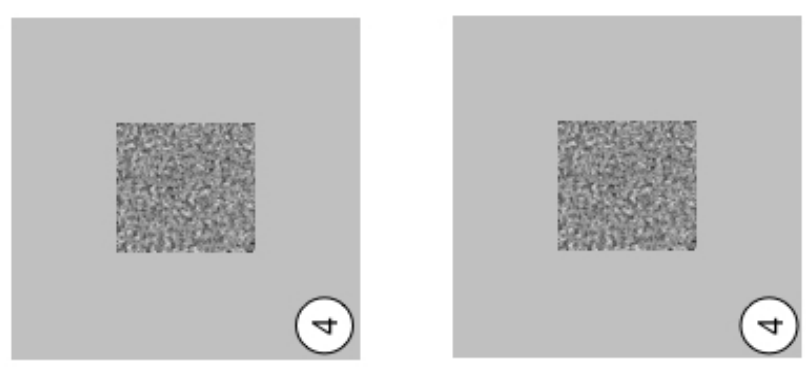

전

霆

的

क

흐ㅇㅝㅡㅁ

बक

흠

(

(
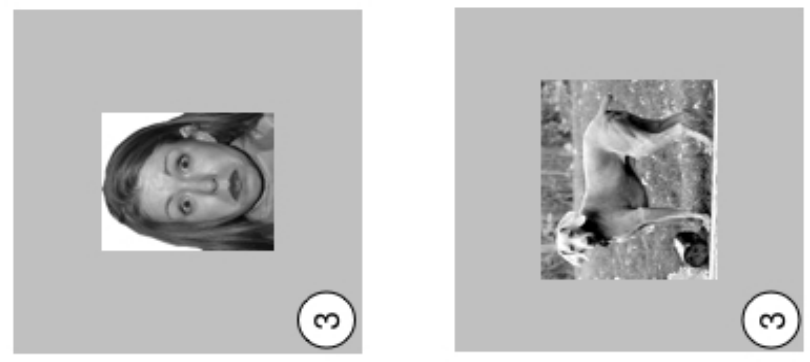

플

क 담

我志

क⿺⿻一⿰冫⿰亅⿱丿丶丶⿱⿰㇒一乂

늠

它饮

N

堽㐘
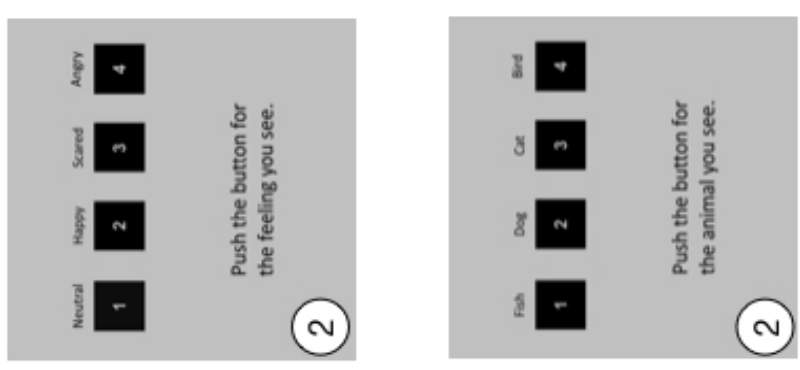

ธक ळ

응

잉

옹

믐ำ

들 뜰 땡

잉ㄷ

는 능

중 흔

눈요

웅
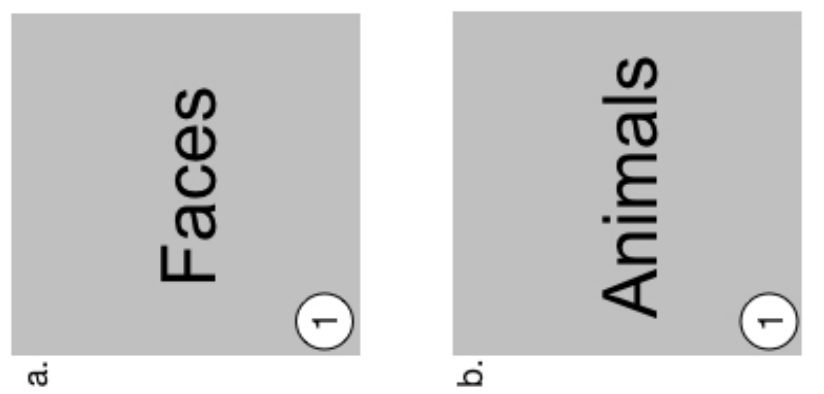

흔

흔 뽀

농

สิธ

త్త

मे 흥

(1)

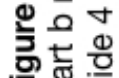

훈 뜽 음 
paracingulate cortex, superior temporal sulci, and the temporal poles bilaterally were found to be consistently activated in Theory of Mind tasks. It was also hypothesized that due to their roles in ToM, that the amygdala and orbitofrontal cortex may also be areas of activations. In 1990, Brothers theorized that the orbitofrontal cortex (OFC), superior temporal gyrus (STG), and amygdala comprised a network of neural regions that made up the "social brain" (Brothers, 1990). Then in 1994, Baron- Cohen and Ring attempted to test this theory and proposed that not only should the orbitofrontal region be activated during ToM, but that any damage to this area would yield subtle impairments in ToM. Baron-Cohen et al. are the only ones to have found increased neural activations in the amygdala (1999) and orbitofrontal regions (1994) while performing a Theory of Mind task in an fMRI study. Gallagher and Frith (2003) also observed deficits in executive function and attention in participants with frontal lesions while performing ToM tasks, which may support Baron-Cohen et al.'s theory. In more recent studies, it has been found that there is reduced network homogeneity within the default mode network (DMN) of participants with ADHD than with age-matched controls. The study found that there was decreased functional connectivity between the precuneus and other DMN regions in the ADHD participants (Uddin et al., 2008).

The default mode network (DMN) comprises the medial prefrontal cortex (mPFC), posterior cingulate cortex (PCC), precuneus, and posterior parietal regions, and serves as a baseline or control network that is engaged while a person is resting and not performing a cognitive task. This network is suppressed when a goal-directed or attention-demanding cognitive task is introduced to the participant (Raichle et al., 2001). If the DMN is not suppressed, then momentary 

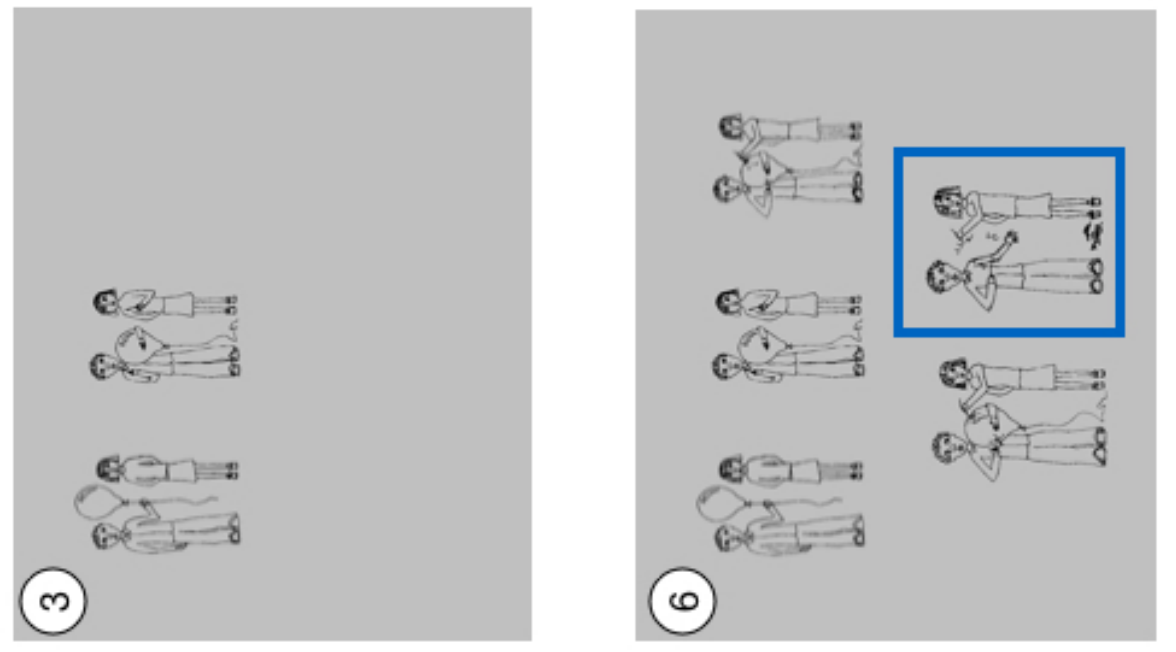


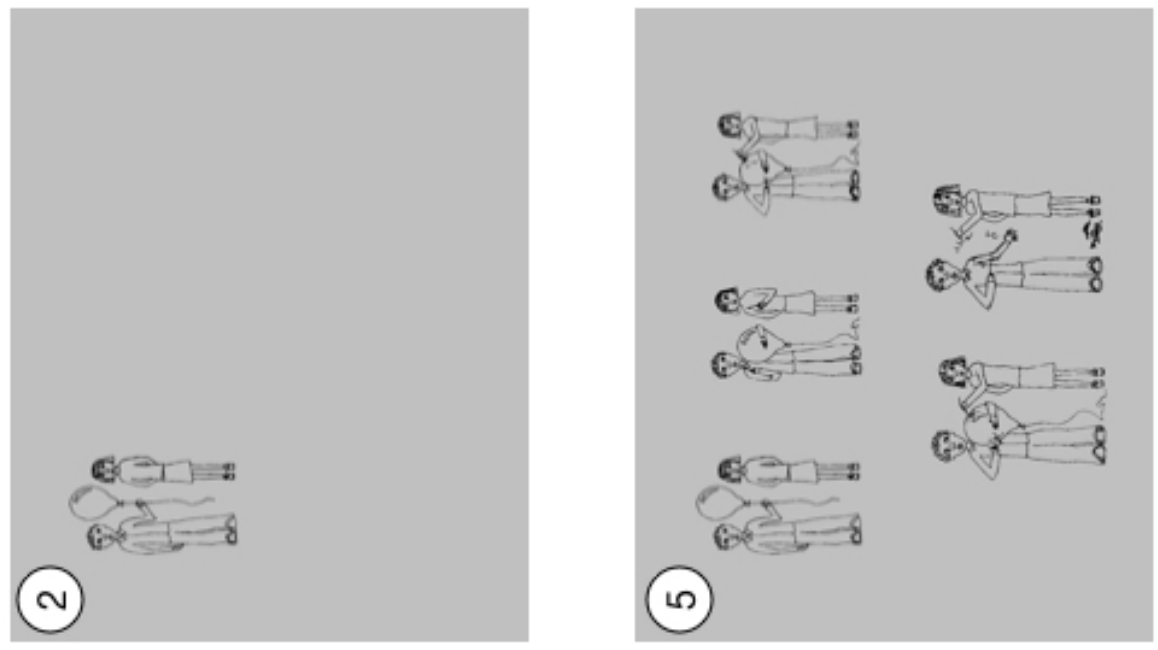

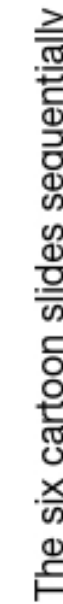

호옳

으음

믇ㅇㅇㅇ
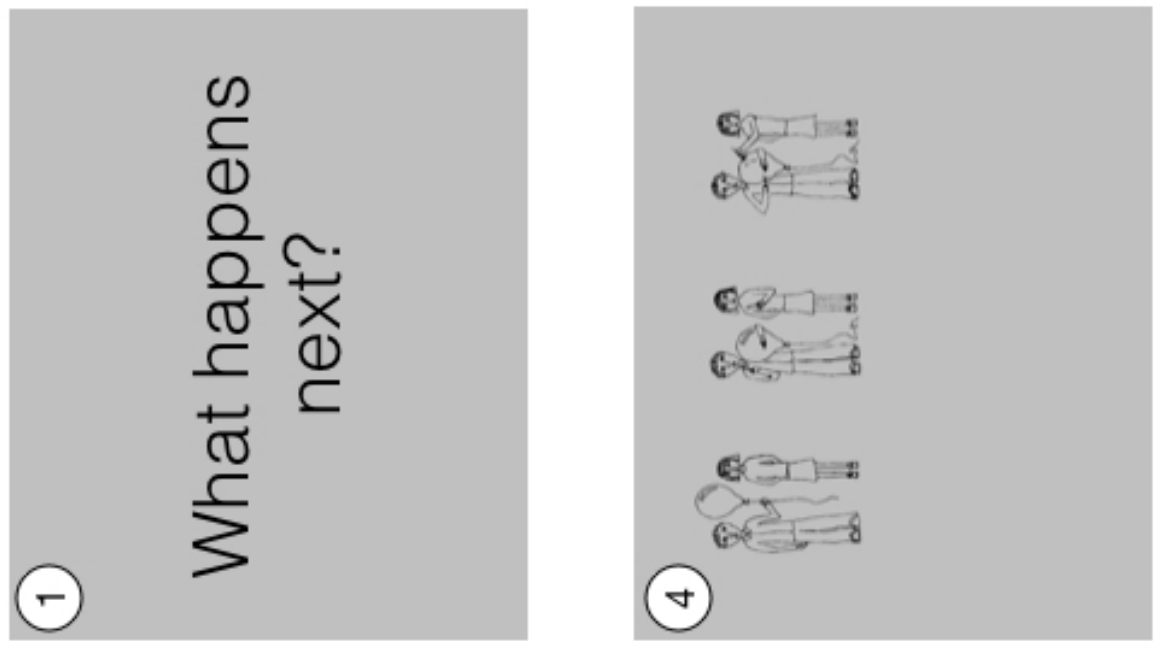

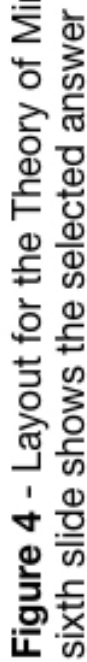


lapses in attention have been known to occur (Weissman et al., 2006). A study by Castellanos et al. (2008) suggested that the precuneus may be the site of dysfunction in ADHD due to the decreased functional connectivity in between the dorsal anterior cingulate cortex (dACC) and precuneus/PCC regions, and with the precuneus and other DMN components. These default mode, amygdala, and frontal regions may help distinguish a network (i.e., identify the neural regions that may be implicated with social and behavioral deficits) involved with social cognition in an atypically developing child population with ADHD.

In a review article, Mitchell and Phillips (2015) observed a relationship between emotion perception and Theory of Mind constructs and neuroanatomy. The two constructs have been viewed differently in the psychological literature (i.e., some papers say the two are related, others say that they are separate abilities, while others believe that emotion perception is a precursor to theory of mind). However, the findings from the neuroimaging literature are fairly convergent, and show overlapping regions in the temporal and frontal regions in both tasks in healthy participants, including the prefrontal cortex, the temporal pole, and the temporo-parietal junction. Differences in activated regions arise due to variability in the task design (e.g., affective versus cognitive in Theory of Mind tasks) and the valence of emotional cues. These connections between the tasks confirms that they may be used together test social and behavioral cognitive deficits in children with ADHD. 


\section{Thesis Objectives}

The research presented in this thesis is novel and is intended to provide new information, while reproducing previous scientific findings (i.e., default mode, amygdala, and frontal activations). Neural activations associated with the Facial Emotion Perception Task (FEPT) and Theory of Mind (ToM) tasks have not yet been investigated in children with ADHD. During the FEPT and ToM tasks, we predicted observation of activity in neural regions such as the amygdala, prefrontal cortex, and insula, as these regions have been previously found in healthy typically developing children. Our objective was to contrast two conditions for each task: facial expressions and animal types in FEPT and physical causality (i.e., cause and effect scenario) and affective Theory of Mind (i.e., scenarios that depict a character's emotional story) in ToM. When analyzing the behavioral data from the Facial Emotion Perception Task, we expected to find that the participants will take longer to decide which emotion they saw and will have more difficulty identifying Fear, Angry, or Neutral expressions. For the ToM task, we hypothesized that the participants will take longer to choose the

ending during the affective ToM story cartoons than the physical causality cartoons, and will accurately predict the ending to the physical causality questions more often than the affective ToM questions. These findings are targeted at providing insight into a possible neural anomaly or cognitive deficit in social impairment in ADHD. 


\section{Methods}

\section{A. Participants}

Participants from this study aged 8-12 were recruited from Florida International University's Summer Treatment Program (FIU; STP), which serves to aid children with ADHD through cognitive behavioral treatment programs (Pelham Jr

and Hoza, 1996). Children enrolled in the FIU STP have undergone a rigorous diagnostic procedure and meet DSM-IV criteria for ADHD. The participant's parents provided written informed contest, while the participants provided written informed assent. Both forms were in accordance to guidelines set by the Institutional Review Board (IRB; IRB-15-0109) at FIU. The parents were also asked a series of questions to attain a basic background of each participant (i.e., age, gender, race, ethnicity, etc.) and to screen out children who were unfit to participate this study due to MRI safety precautions (i.e., pacemakers, any metal in the body, pregnant, etc.). After the extensive screenings, ten participants were approved and enrolled in the neuroimaging study.

\section{B. fMRI Data Acquisition and Experimental Tasks}

A GE MR750 3.0T MR Scanner (University of Miami, Coral Gables, FL) with a 32-channel head coil was used to acquire structural (anatomical) and functional images of the participants' brains. The high resolution three-dimensional T1weighted (structural) images were acquired for anatomical reference [repetition time (TR), $2500 \mathrm{~ms}$; echo time (TE), $4.38 \mathrm{~ms}$; flip angle (FA), $8^{\circ} ; 1.0 \mathrm{~mm}$ isotropic voxels]. Functional $\mathrm{T}^{*}$-weighted images were acquired using a single-shot 
gradient-echo echo-planar pulse sequence (TR, 2000 ms; TE, 27 ms; FA, 78; $3.44 \mathrm{~mm}$ isotropic voxels). Earplugs and headphones were used to attenuate scanner noise to protect participant hearing.

During the functional scans, participants were asked to complete two mental tasks, the Facial Emotion Perception Task (FEPT) and the Theory of Mind (ToM) task. The tasks were created using the psychology software program E-Prime, and contained instructions before starting each task. Before the participants performed the tasks inside the MRI scanner, they were trained outside the scanner at a computer desk and then practiced inside a mock scanner (i.e., replica of the scanner, but without the magnetic field or components). This ensured that the participants were confident and capable of performing the tasks in the MRI environment.

During the first task, Facial Emotion Perception Task (FEPT), participants viewed 150 emotional faces and were instructed to correctly identify the facial expression (e.g., neutral, happy, fear, angry) using a four-button box (i.e., a button corresponding to each choice). During control trials, participants viewed pictures of animals and were asked to indicate the type of animal shown (e.g., dog, cat, bird, or fish). This task was adapted from Weisenbach et al. (2012). Data were collected for the FEPT across two runs. Each run comprised of three face blocks and three animal (control) blocks, and each block lasted for 28 seconds and presented five images for 0.6 seconds each (Figure 5). Each image of the set was followed by a 0.1 second visual mask to prevent visual afterburn phenomena (i.e., the "burning" of an image in one's mind, even after the image is no longer 
present) and a response which showed the finger mapping of the emotions/animal types presented. To ensure unbiased results, the faces were manually randomized to balance gender, emotion, and race, and there were also four versions (i.e., mappings) of each run to mix the order of the emotions on the response slide. This task consisted of two runs, each lasting approximately 5 minutes.

During the second task, Theory of Mind (ToM), participants viewed a series of cartoons revealing a story (cartoon vignette) and were instructed to indicate the correct ending of the story using a button box. This task was adapted from Sebastian et al. (2012) and contained two types of stories: affective ToM (aToM) and physical causality (PC). The affective ToM stories required the participant to infer what a story character was feeling, while the physical causality stories displayed cause and effect situations. This task was broken down into two runs (11 minutes total) of ten blocks, each block containing two story cartoons (Figure 5); either two physical causality story cartoons (controls) or two affective Theory of Mind story cartoons (stimulus). There were a total of twenty story cartoons (i.e., ten aToM and ten PC). The story cartoons were presented in six slides, and lasted for 21 seconds. The first slide gave the instruction of "What happens next?," and was displayed for 3 seconds. The next three frames showed the "story" cartoons, which were sequentially added (3 seconds each; 9 seconds total). A choice endings slide was then presented for 8 seconds and contained the three story images in smaller sizes, along with the two possible endings (i.e., correct and incorrect endings). A blue box around the selected choice cartoon 
appeared after the participant's choice ending response had been made via the button box. Succeeding each story cartoon was an interstimulus interval of one second and a fixation cross.

\section{Data Analysis}

The fMRI data were analyzed using AFNI (Analysis of Functional Neurolmages) (Cox, 1996) and FSL (FMRIB Software Library) (Woolrich et al., 2009; Smith et al., 2004; Jenkinson et al., 2012). These software programs were used in tandem to normalize the brain images to a standardized template (MNI152 template; Collins et al., 1994), and identify which brain regions were activated during the tasks. Specifically, FSL's fMRI Expert Analysis Tool (FEAT; version 6.00) was first used to perform the following pre-processing operations: motion correction, non-brain removal through FSL's Brain Extraction Tool (BET), spatial smoothing with a 5mm FWHM Gaussian kernel, high-pass temporal filtering (to remove artifacts associated with scanner drift), and linear registration to MNI space. The first 5 volumes were discarded to eliminate magnetization saturation effects. Then, to isolate the neural regions participating in each task, a General Linear Modeling (GLM) analysis was performed on the pre-processed functional data set to model the contrasts of Faces $>$ Rest, Animals $>$ Rest, and Faces $>$ Animals for the FEPT task and Affective ToM > Rest, Physical Causality > Rest, and Affective ToM > Physical Causality for the ToM task. A group-level analysis was then performed to identify those regions exceeding statistical significance $\left(Z_{\text {voxel }}>2.3\right.$, $P_{\text {cluster }}<0.05$ ) across all subjects for each contrast. 


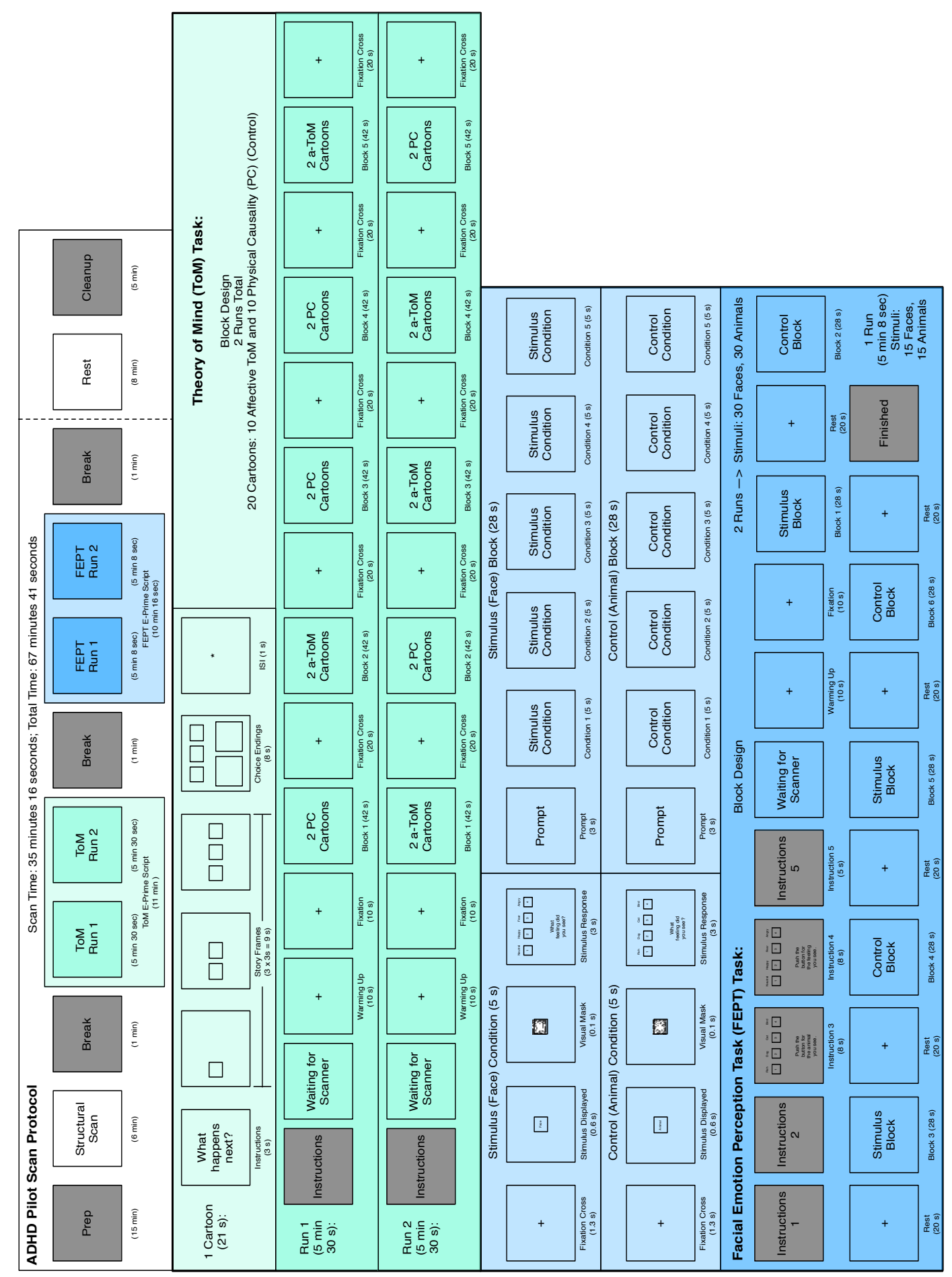

Figure 5 - Scan Protocol Layout illustrating the breakdown of the scanning session and each task. 
After creating and investigating the activation maps that were produced, the behavioral data were analyzed (i.e., response times, selected responses, skipped questions, etc.).

Quantitative group-level analyses were performed to examine response times for the FEPT and ToM tasks. F-tests and t-tests were used to quantify the differences in response times for faces and animals, and physical causality and affective Theory of Mind cartoons. An ANOVA (ANalysis Of VAriance) was also used to assess whether subjects had faster or slower response times to specific types of emotions during the FEPT task (e.g., happy, fear, angry, neutral), and to the specific story cartoons during the ToM task, demonstrating that one emotion/story type takes longer to identify. Also, the percentage of incorrect answers were calculated, and an f-test and t-test were used to determine differences in accuracy between the emotion/story-type categories.

\section{Results}

Out of the ten children recruited and enrolled, four were not scanned due to scheduling conflicts, and one participant's data was not used due to excessive motion (determined by a large framewise displacement; a variable that measures the movement of any frame relative to the previous frame in order to quantify head motion). Framewise displacement is especially important in this study as we collected imaging data from young children with ADHD, who exhibit excessive motion in the scanner. The remaining five participants' data were found suitable 
for analyses as their head motion, predominantly remained under the standard 1 $\mathrm{mm}$ threshold used for task-based functional MRI (Figure 6).

\section{A. Task 1 - Facial Emotion Perception Task Behavioral Results}

The Facial Emotion Perception Task resulted in the least head movement, as compared to movement acquired during the ToM task. The FEPT task had a more uniform and centered layout, which did not cause a shift in attention/gaze from the center of the screen. In comparison, the ToM task had a layout that changes every few slides. For example, after the first slide (centered), three story cartoons were sequentially introduced in the top half of the screen. This first story cartoon caused a shift in visual gaze, which in turn caused an upward head shift. After the story cartoons were visible, the choice endings cartoons appeared on the bottom of the screen. This new directional appearance of an object caused a downward head motion from some of the participants. It is due to these uncontrollable and undesired head movements, that one of the participant's data was not able to be used. Four of the five participants retained more than $94 \%$ of the data at the standard $1 \mathrm{~mm}$ threshold for task-based functional MRI (Figure 6). When comparing to a more rigorous threshold $(0.5 \mathrm{~mm})$, more than $73 \%$ off the data were below this quantity.

Additional behavioral data is provided in Table 1 to summarize the emotional responses made during the FEPT task. Overall, the participants were able to correctly identify $75 \%$ of the facial expressions, and only $7 \%$ of the expressions were skipped. It is apparent that the participants were best able to correctly 
categorize a happy emotion ( $83 \%$ correctly identified), and in the fastest time (857ms = mean response time; Table 2a). The other three emotions (anger, fear, and neutral) accounted for $21 \%$ of the total incorrect responses. When comparing the mean response times, happy and angry had the fastest mean response times, while neutral and fearful on average (almost equally) took longer time to identify the emotion presented and key in an answer (Table 2a). Nonetheless, these mean response times for face stimuli were much longer than those for the animal stimuli. The average response time for emotional responses as a whole was $1122.03 \mathrm{~ms}$, while animals required an average of $25.00 \mathrm{~ms}$ for a response.

Table $2 \mathrm{~b}$ shows the t-statistics and the $\mathrm{P}$ values for the mean accuracy (acc.) and response times of the six emotion pairs (neutral-happy, neutral-angry, neutral-fear, angry-happy, fear-happy, and fear-angry). The emotion pairs all had equal variance when looking at the accuracy percentages and the response times, except for neutral-happy and fear-happy in the response times. Four emotion pairs (neutral-happy, neutral-angry, fear-happy, and fear-angry) were observed to have response time means that differed significantly. Those pairs can be seen by the dagger in Table 2 .

The last statistical tool used to analyze the data was an ANOVA table for the accuracy $(\%)$ and the response times. The results indicated that the means of the accuracies were equal, but not for the response times of the emotions. These results confirm the previous results obtained in Table $2 \mathrm{~b}$. Taking a closer look at the statistical findings of the accuracy percentage ANOVA, it was found that the 


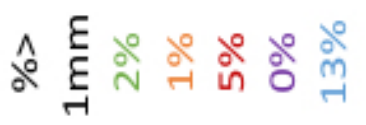

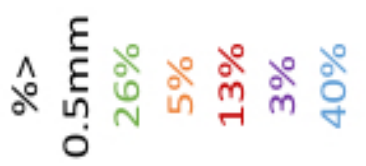

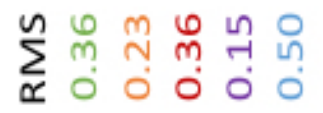

돌

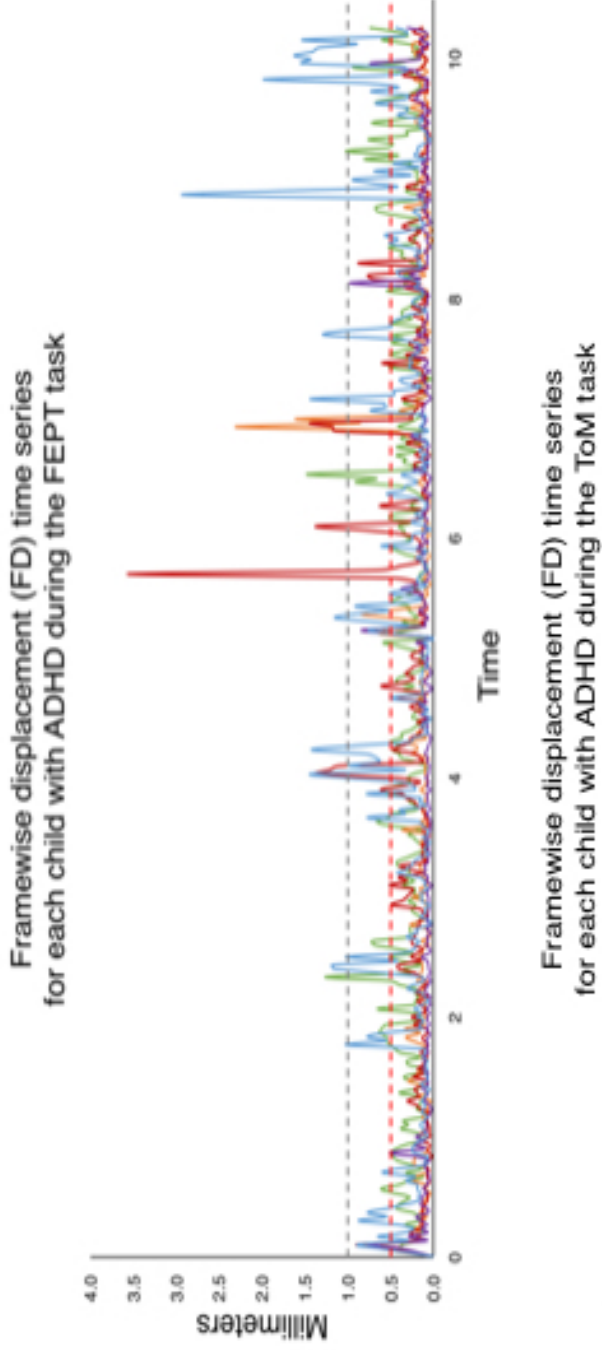

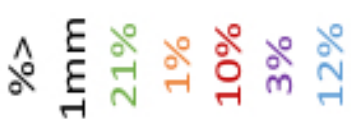

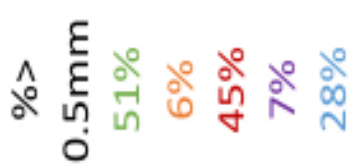

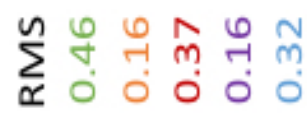

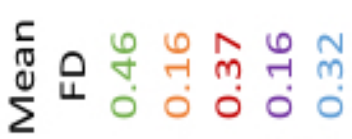

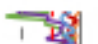

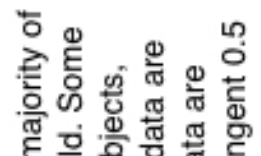

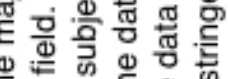

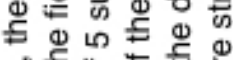

३ั.

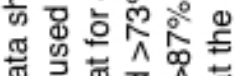

तi 궁

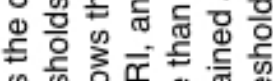

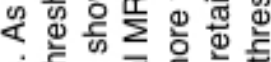

की

क्ष

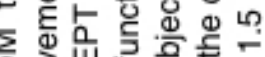

은

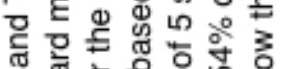

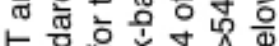

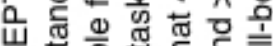

世

ब

응

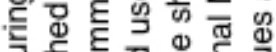

흐의의

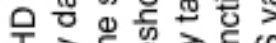

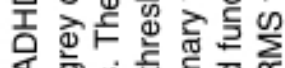

< 항

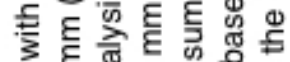

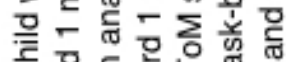

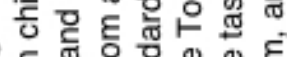

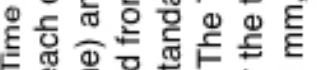

드

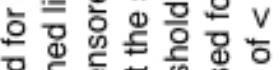

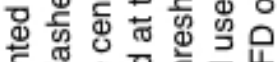

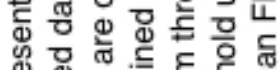

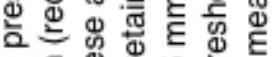

Фह है

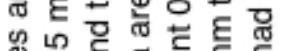

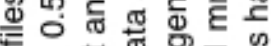

은 눙 응

혀잉

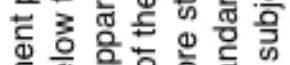

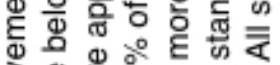
产 。

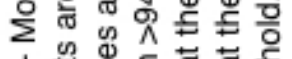

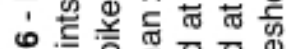

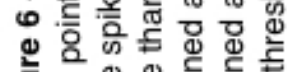

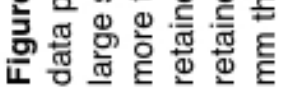


probability that the differences among the four emotions (neutral, happy, angry, and fear) due to chance alone was 0.2665 . This indicates that the emotional expressions affected the accuracy percentages. However, the results of the response time ANOVA show that with the same sample size of 150 , the probability that the differences observed among the emotions were due to chance is 0.0020 . This low P-value denotes that the emotion types had no effect on response times.

After examining all the results, it was still unclear if the participants were confusing specific emotional pairs. For this reason, Figure 7 was created to illustrate the error rates for the emotion relationships. This figure shows the frequency that one emotion was selected in place of another emotion, and vice versa. For example, when looking at the responses to the happy expression, it was observed that the participant three times incorrectly selected "neutral," twice incorrectly selected "fear," and once skipped the response. Putting these observations in a graph indicated that although there was no (statistically) evident emotion that was harder for the participants to correctly classify, it appeared that fear and anger were the two emotions that were the most challenging for the participants (i.e., incorrect and skipped selections). In addition to this appendaged data analysis, the prospect of a possible reflex or selective guessing technique was investigated. This possible reflex or suggestive guessing is analogous to a student choosing " $\mathrm{C}$ " as their go-to answer when unsure about a question. However, the data did not suggest a clear correlation to a particular response key or emotional expression, and the twenty-six incorrect answers. 


\begin{tabular}{|c|c|c|}
\hline $\begin{array}{l}\bar{\delta} \\
\frac{0}{2} \\
\frac{0}{\bar{y}} \\
\circ \\
\circ\end{array}$ & $\stackrel{\circ}{\circ} \stackrel{\circ}{\circ} \stackrel{\circ}{\circ}$ & \\
\hline $\begin{array}{l}\bar{d} \\
\frac{0}{0} \\
\frac{0}{\bar{s}} \\
* \\
*\end{array}$ & $\begin{array}{llll}-\infty & \infty & N\end{array}$ & \\
\hline 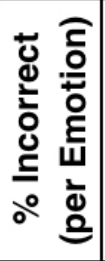 & 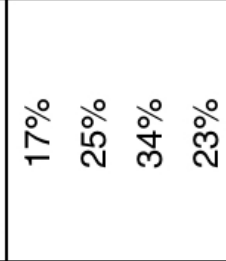 & \\
\hline 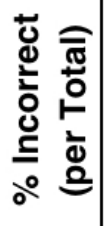 & 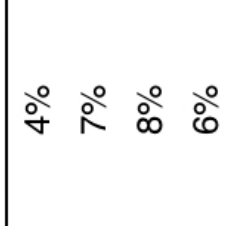 & \\
\hline 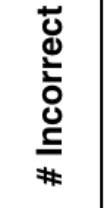 & $0 \div \cong \sigma$ & \\
\hline 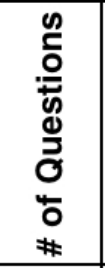 & 요 q 占 q & \\
\hline 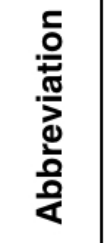 & 至飞岁岩 & \\
\hline 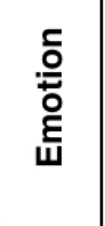 & 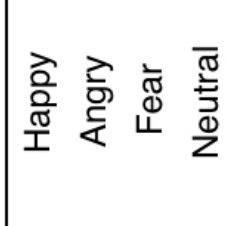 & \\
\hline
\end{tabular}




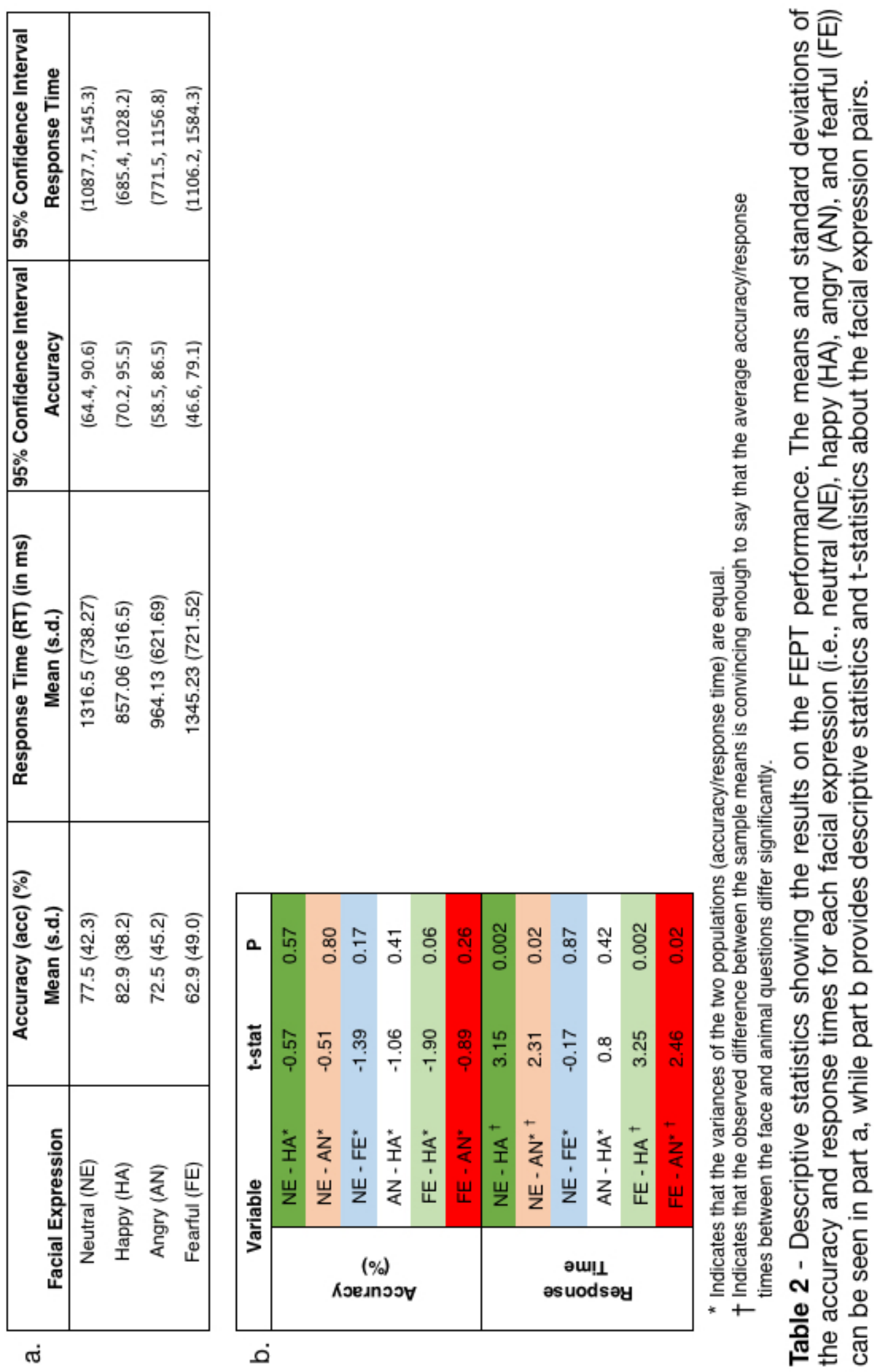




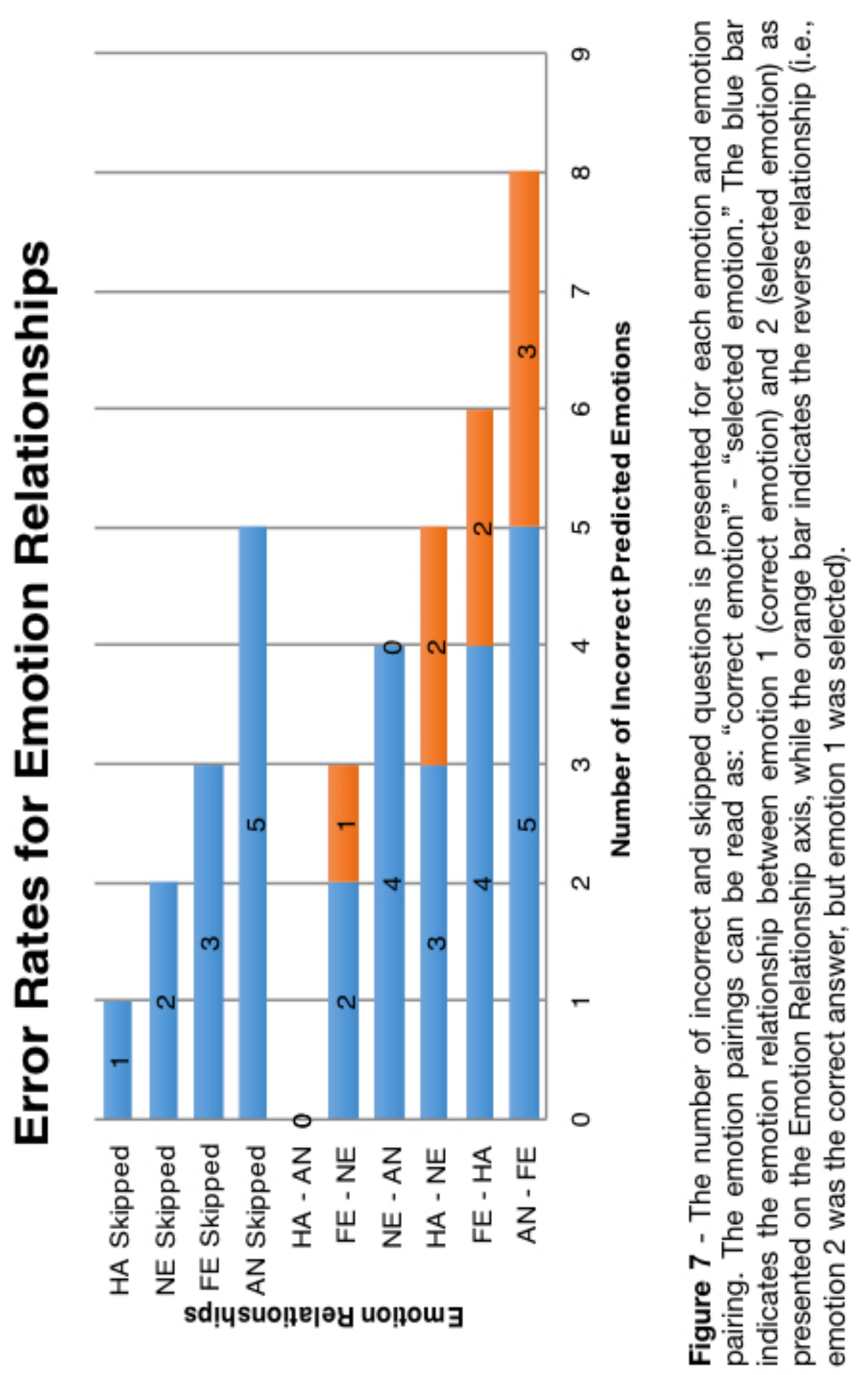




\section{B. Task 2 - Theory of Mind Behavioral Results}

The Theory of Mind task was observed to induce greater head motion as compared to the emotion task. As mentioned earlier, this was caused by the presentation and placement of the story and choice endings cartoon images. Figure 6 illustrates how the head motion (framewise displacement) data was found to have more than $87 \%$ of the points lie below the $1 \mathrm{~mm}$ threshold, and more than $54 \%$ of the points retained at the more stringent $0.5 \mathrm{~mm}$ threshold for four of the five participants. Tables 3 and 4 show the descriptive statistics regarding the Theory of Mind task. It was observed that the participants performed better on this task than on the Facial Emotion Perception Task, but took longer choosing the correct ending to the story (greater than $73 \%$ accuracy; between three and four seconds to respond). The f-tests quantified that the variances for the accuracy and response times of the affective Theory of Mind (aToM) and physical causality (PC) cartoons were equal. This is not surprising as the number of story endings predicted incorrectly (out of 50) for PC and aToM were 13 and 11, respectively. The t-tests indicated that the difference between the response times means was convincing enough to state that the average response times differed significantly between the aToM and PC cartoons. The ANOVAs for the accuracy and response times demonstrated that the means were equal for the two types of cartoons. The P-values however differed. The P-

value of the accuracy $(0.6436)$ revealed that the types of cartoons affected the accuracy percentages, while the P-value of the response times $(0.0429)$ showed that the types of cartoon had no effect on the response times (Table 4b). 




\section{Facial Emotion Perception Task Imaging Results}

For the Facial Emotion Perception Task, significant activation for the Emotional Faces $>$ Rest contrast was found mostly in posterior regions, including lingual gyrus, fusiform gyrus, cuneus, and cerebellum. The insula (left), frontal gyrus (superior, middle, and inferior), dorsolateral prefrontal cortex (DLPFC), anterior cingulate, and pre- and postcentral gyri were also significantly activated. Figure 8 shows these frontal, parietal, occipital, and temporal lobe activations.

When viewing and discriminating the animals (when compared to rest), activation was observed in several of the same posterior regions, but to different extent (i.e., volume). The left pre- and postcentral gyri, along with the left insula, cerebellum, fusiform gyrus, and lingual gyrus seemed to have a greater extent activated during the visualization of animals (i.e., larger volumes of regions of activation as compared to Faces > Rest; Figure 9). However, the anterior cingulate and frontal gyrus appeared to be activated to a lesser extent (i.e., smaller volumes of regions of activation as compared to Faces $>$ Rest).

When contrasting the discrimination of facial expressions and animals (i.e., Faces $>$ Animals), some of the regions that were in common did not appear, as expected. Regions of the insula, pre- and postcentral gyri, and cerebellum remained (i.e., were not subtracted out in the contrast analysis), with the additional observation of the posterior cingulate, cingulate gyrus, temporal gyrus, and the parahippocampal gyrus (Figure 10). The reverse contrast (i.e., Animals > Faces) displayed an uncanny resemblance to the Faces $>$ Animals contrast in the sense that similar regions were activated. The differences, however, can be 
observed in the extent of the activations (i.e., the volume of each region), and that the left pre- and postcentral gyri were not present (Figure 11). 


\section{FEPT Task: Emotional Faces $>$ Rest}



Precentral and

Postcentral Gyri

$z=58$

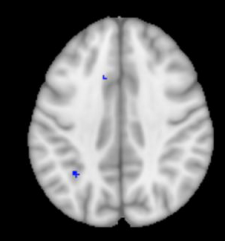

$z=38$

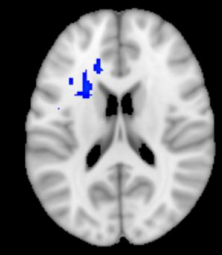

$z=18$

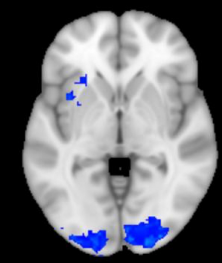

$z=-2$

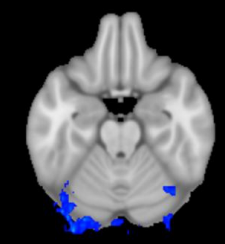

$z=-22$

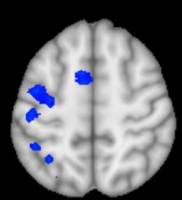

Frontal

Gyrus
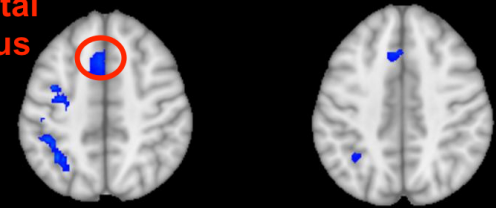

Anterior


Cuneus
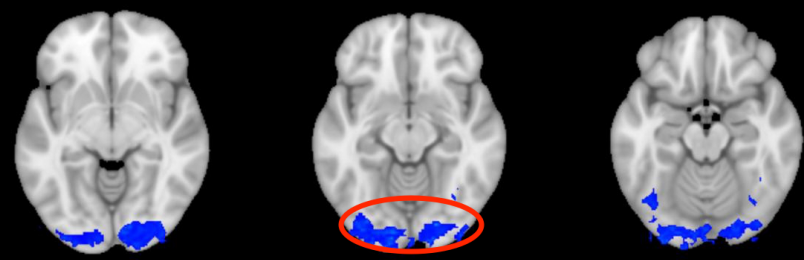

Fusiform and

Lingual Gyri
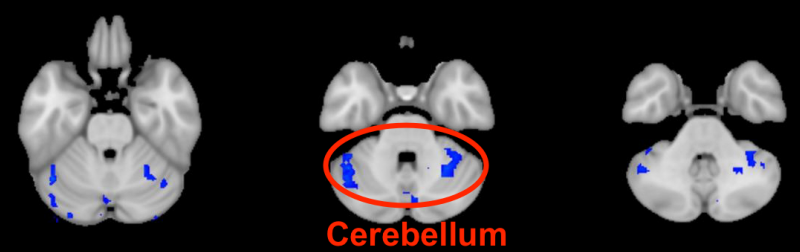

\section{$2.3 \quad z \quad 9.898$}

Figure 8 - The regions of activation for the FEPT task while viewing the emotional faces versus while at rest. 


\section{FEPT Task: Animals > Rest}

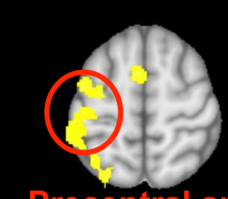

Precentral and

Postcentral Gyri $z=58$

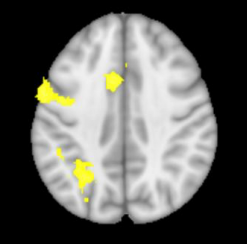

$z=38$

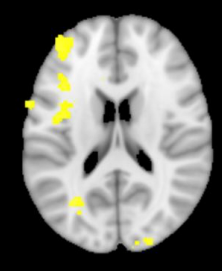

$z=18$

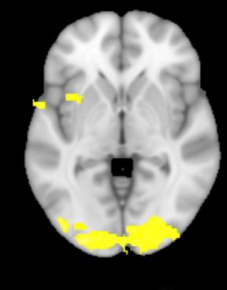

$$
z=-2
$$

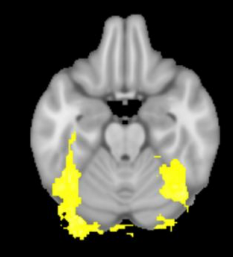

$z=-22$
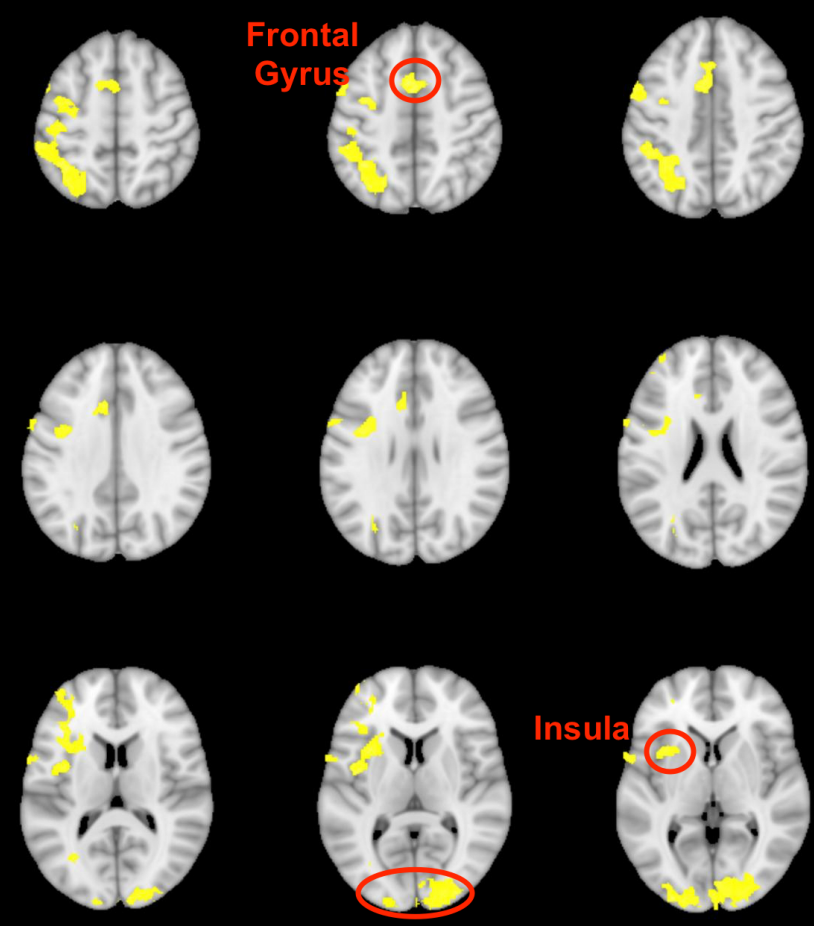

Cuneus


Fusiform and Lingual Gyri


\section{$2.3 \quad Z \quad 9.933$}

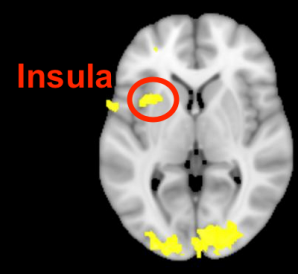

L

Figure 9 - The regions of activation for the FEPT task while viewing animal pictures versus while at rest. 


\section{FEPT Task: Faces > Animals}
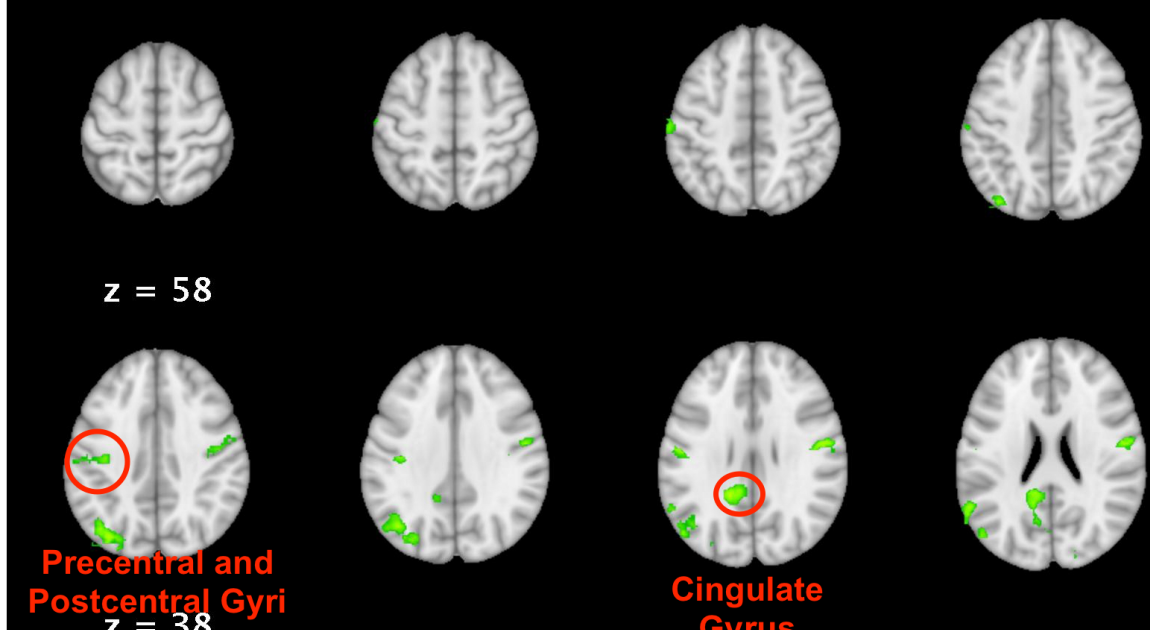

ostcentral Gyr
$\mathbf{z}=38$
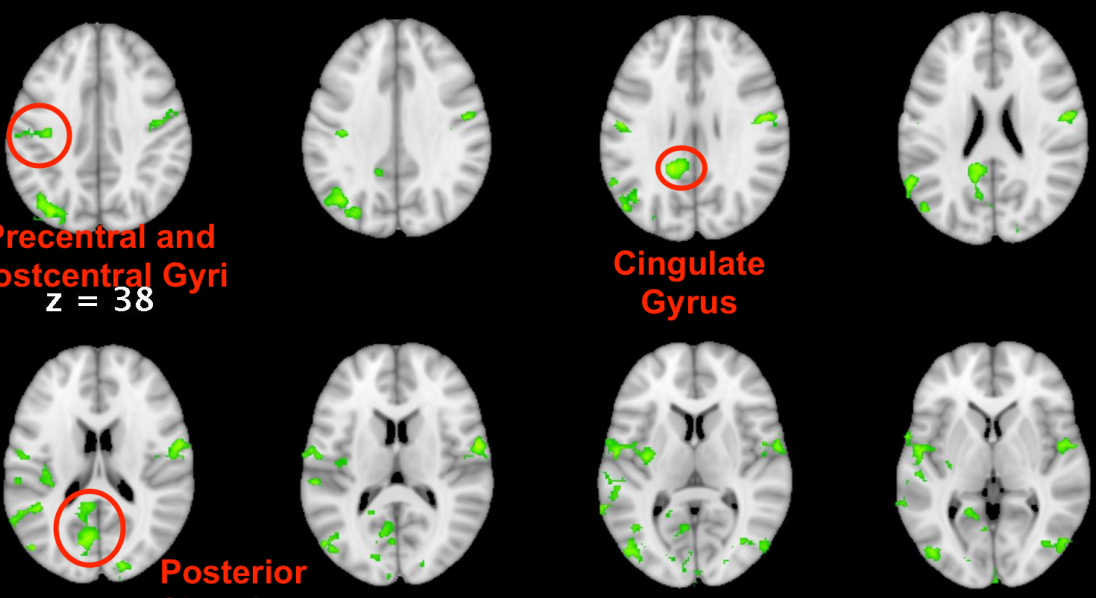

$z=18$
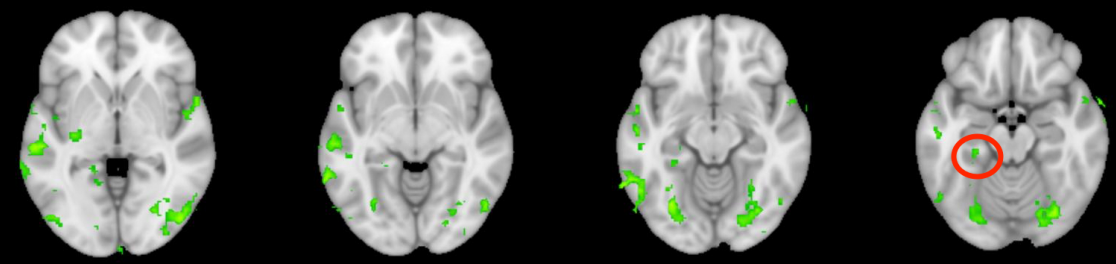

$z=-2$

Parahippocampal Gyrus


$z=-22$

\section{$2.3 \quad Z \quad 5.66$}

Figure 10 - The regions of activation for the FEPT task contrasting viewing the emotional faces and animal pictures. 


\section{FEPT Task: Animals > Faces}
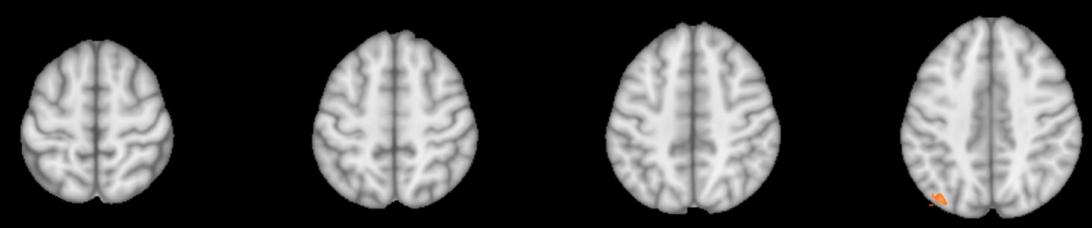

$z=58$
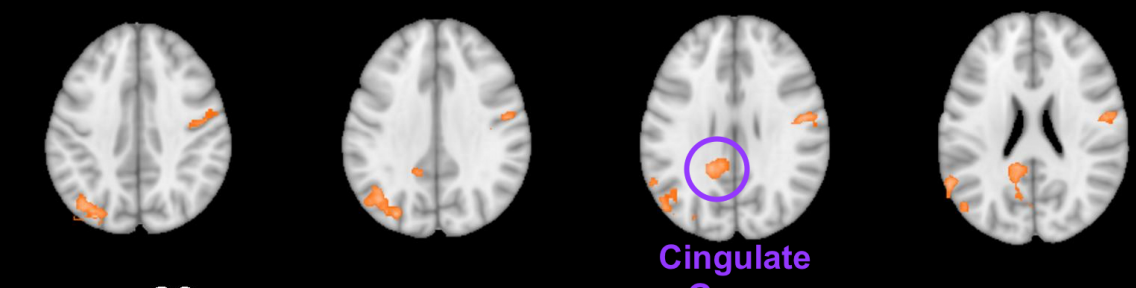

$z=38$ Gyrus
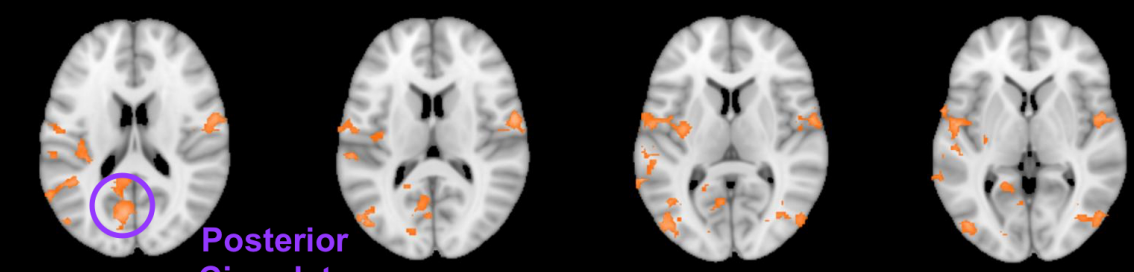

$z=18$ Cingulate
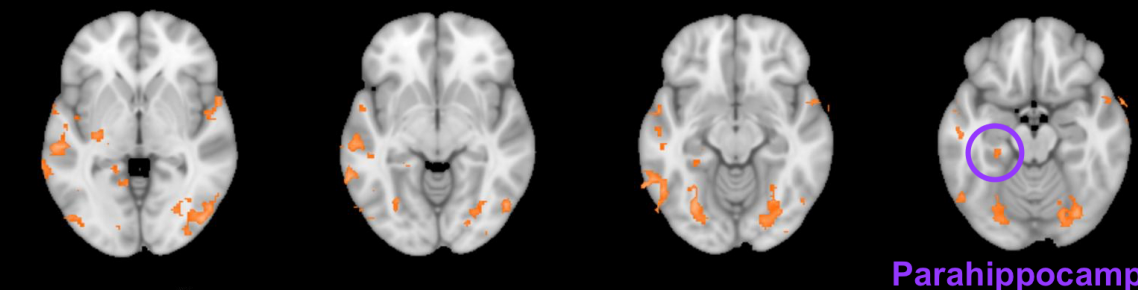

$z=-2$

Parahippocampal Gyrus
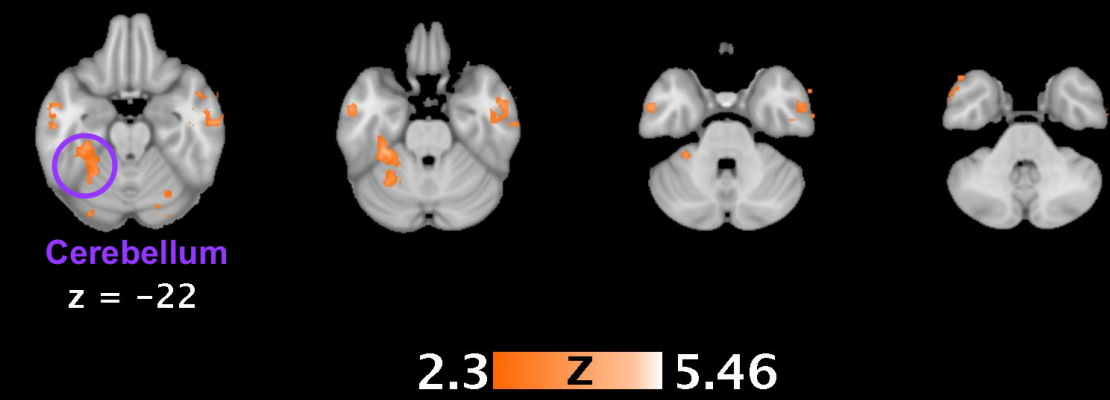

$\mathbf{L}$

Figure 11 - The regions of activation for the FEPT task contrasting viewing the animal and emotional faces pictures. 


\section{Theory of Mind Task Imaging Results}

The activation maps yielded by the Theory of Mind task revealed similar brain regions as the FEPT task. For the affective Theory of Mind (aToM) cartoons (i.e., aToM > Rest), the anterior regions that were activated included the frontal gyrus (middle, superior, and bilateral inferior), dorsolateral prefrontal cortex (DLPFC), and insula (Figure 12). In the posterior aspect of the brain, significant activations in the fusiform gyrus, lingual gyrus, and precuneus were present. The medial aspect of the brain showed the thalamus, insula, parahippocampal gyrus, and pre- and postcentral gyri activated. The middle and superior temporal gyri significantly activated in the anterior and posterior regions of the brain.

The physical causality (PC) cartoons demonstrated activations in mostly almost all of the same regions as the affective ToM cartoons, but to different extents. The middle frontal gyrus and precentral gyrus were the only regions that appeared to have around the same extent of activation between the types of cartoons (Figure 13). It is also worth noting that there were small clusters of activations in the left amygdala during the aToM > Rest $(-20,-9,-14)$ and PC > Rest $(-20,-5,-25)$.

The activation map contrasting the affective ToM and physical causality cartoons (i.e., aToM > PC) showed that there were few brain regions that had a greater extent (or volume) of activation for the affective Theory of Mind cartoons than for the physical causality cartoons. Those regions included the middle and superior temporal gyri and the inferior frontal gyrus (Figure 14). In the reverse contrast of 
PC > aToM, the superior temporal gyrus was also present, but was accompanied by the parahippocampal gyrus and cingulate gyrus (Figure 15). 


\section{ToM: Affective ToM > Rest}
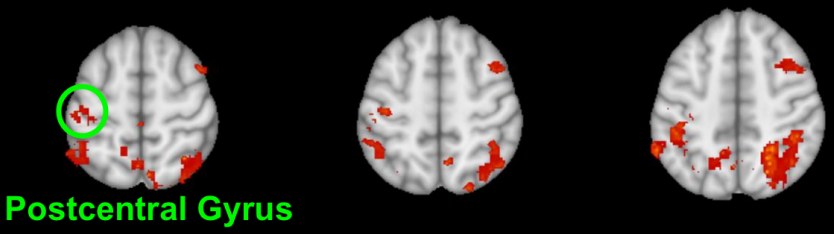

Frontal

$z=58$
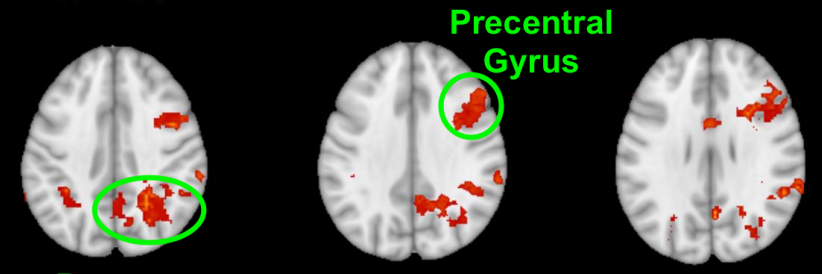

Temporal Gyrus

$$
z=38
$$

Inferior


Frontal Gyrus
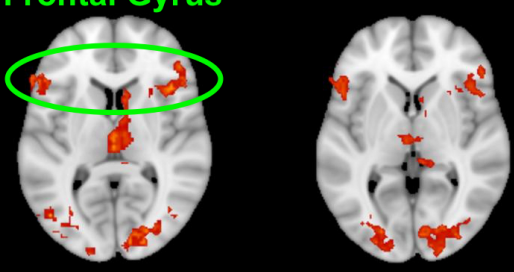

$z=18$

Thalamus
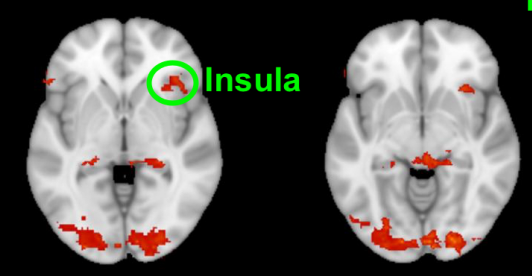

Parahippocampal Gyrus

$z=-2$
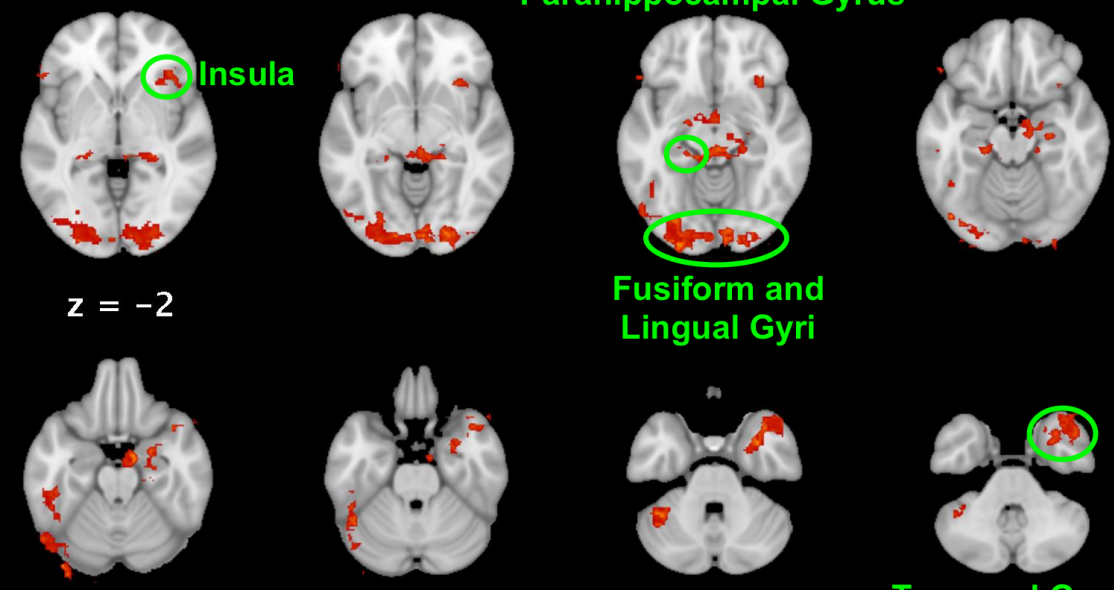

Fusiform and Lingual Gyri

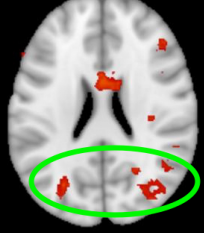

Gyrus

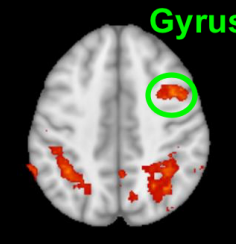

$z=-22$
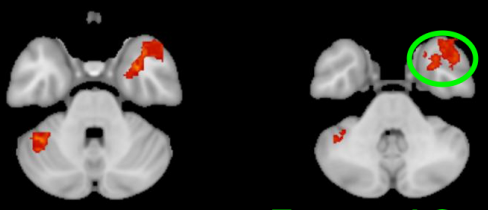

Temporal Gyrus

\section{$\begin{array}{lll}2.3 & \mathrm{Z} & 9.084\end{array}$}

Figure 12 - The regions of activation for the ToM task while viewing affective ToM cartoon stories versus while at rest. 


\section{ToM: Physical Causality > Rest}
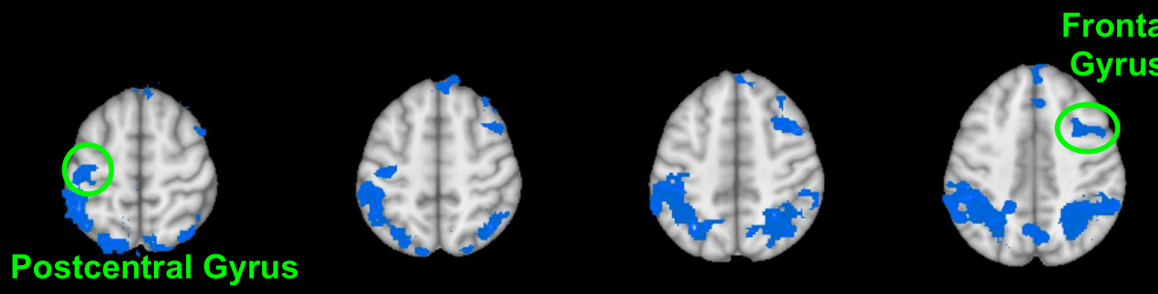

$$
z=58
$$
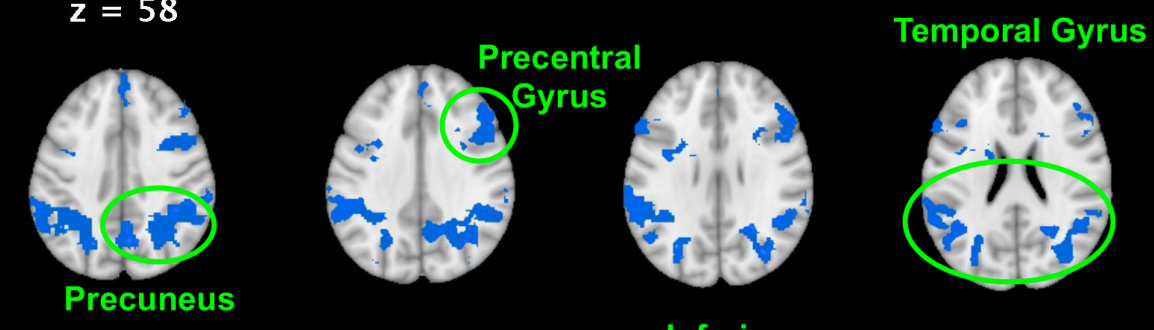

$z=38$
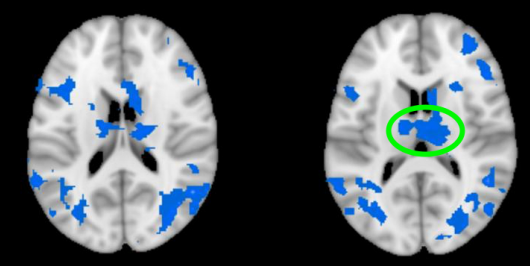

Inferior

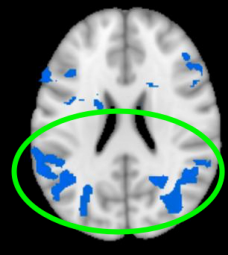

Frontal Gyrus
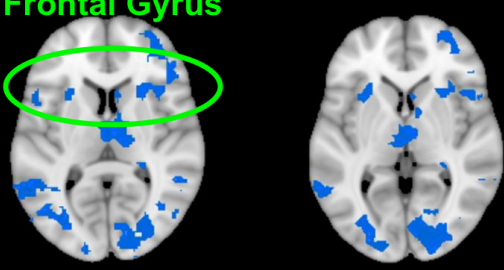

$z=18$

Thalamus
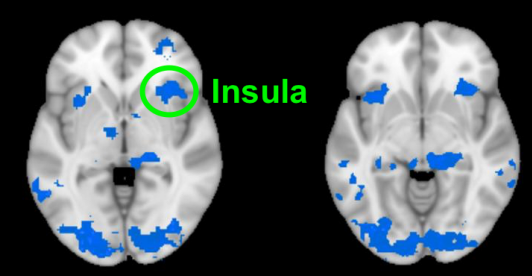

Parahippocampal Gyrus

$z=-2$


Fusiform and Lingual Gyri


$z=-22$

\section{$\begin{array}{lll}2.3 & Z & 9.124\end{array}$}

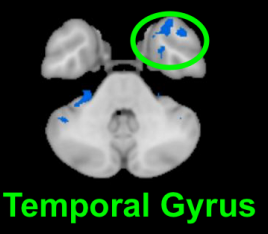

Figure 13 - The regions of activation for the ToM task while viewing physical causality cartoon stories versus while at rest. 


\section{ToM Task: aToM > PC}


$z=58$
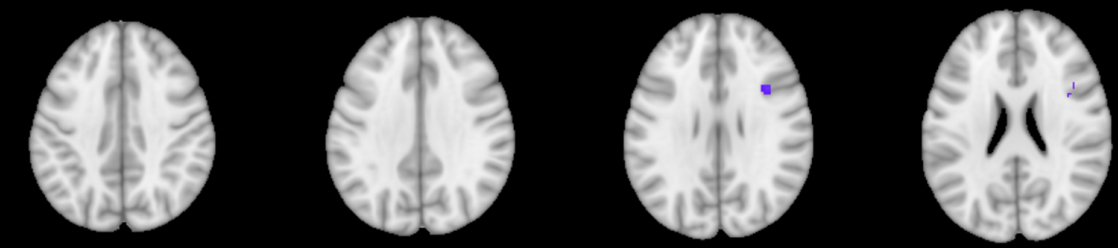

$z=38$
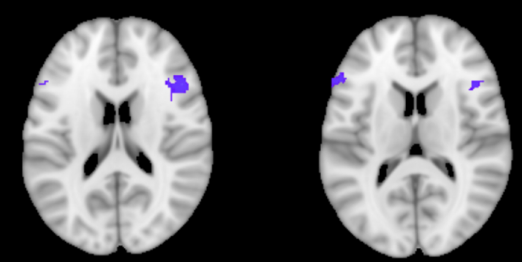

Inferior

Frontal Gyrus
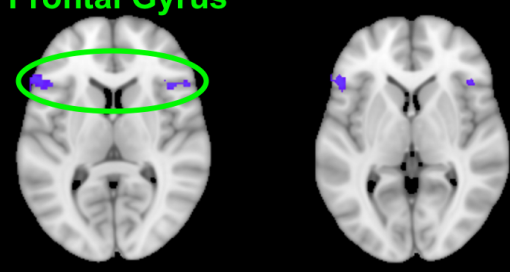

$z=18$
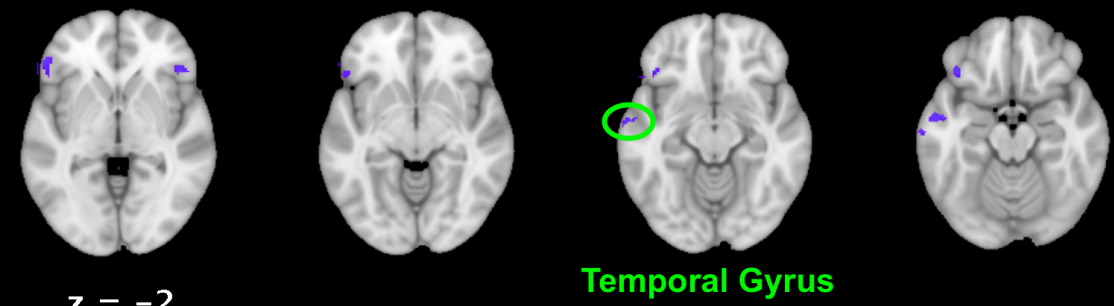

$z=-2$
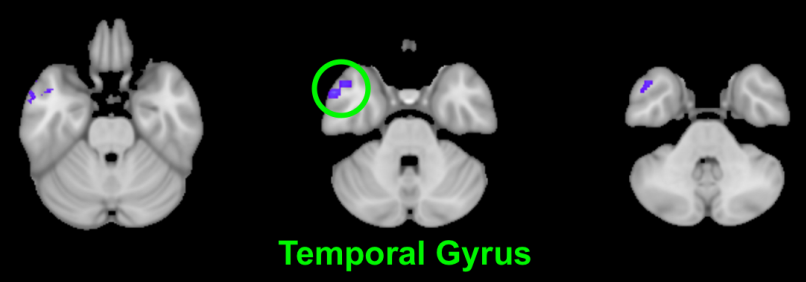

$z=-22$

\section{$2.3 \quad z \quad 5.186$}

Figure 14 - The regions of activation for the ToM task contrasting the visualization of the affective ToM cartoon stories and the physical causality ones. 


\section{ToM Task: $P C>$ aToM}
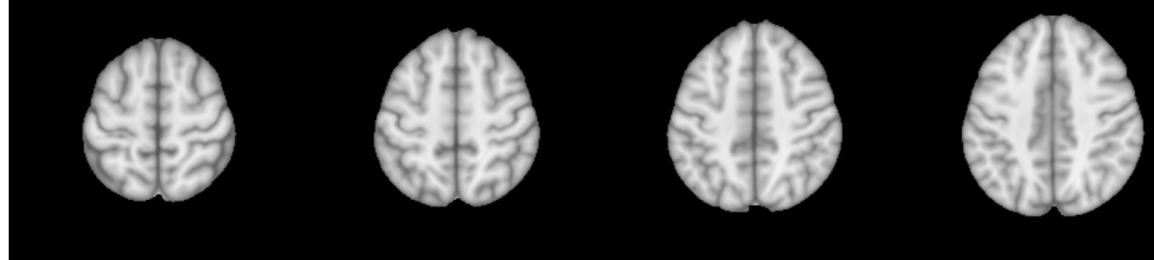

$z=58$

Cingulate Gyrus
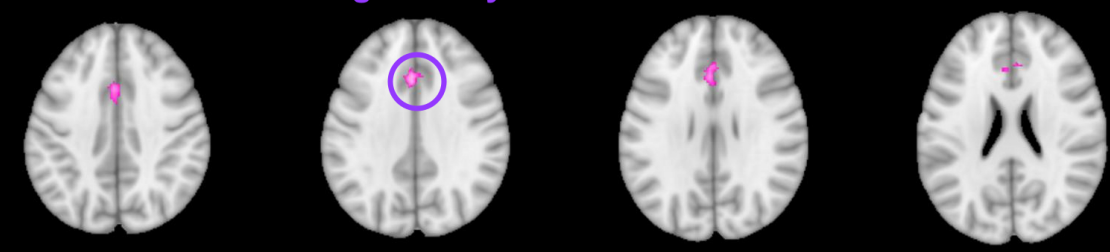

$z=38$
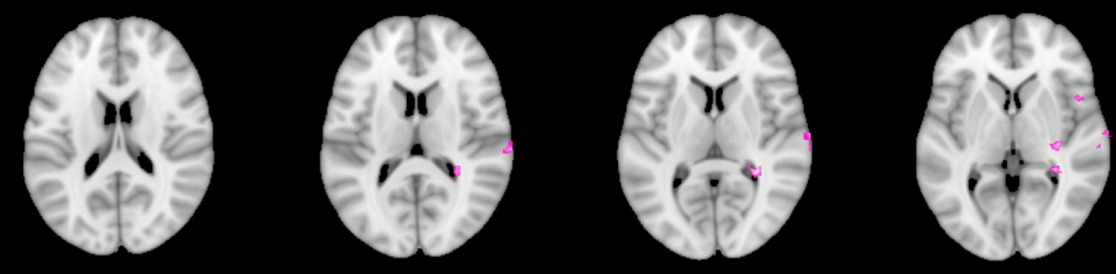

$z=18$
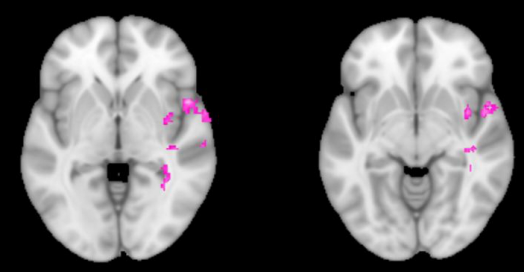

Superior

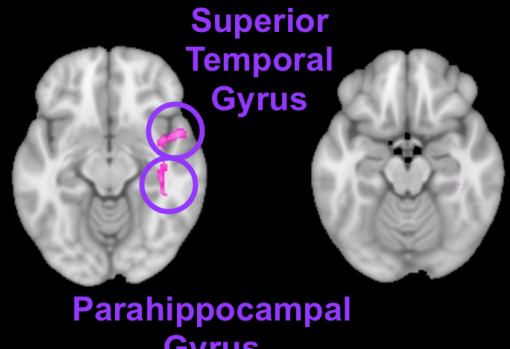

$z=-2$ Gyrus
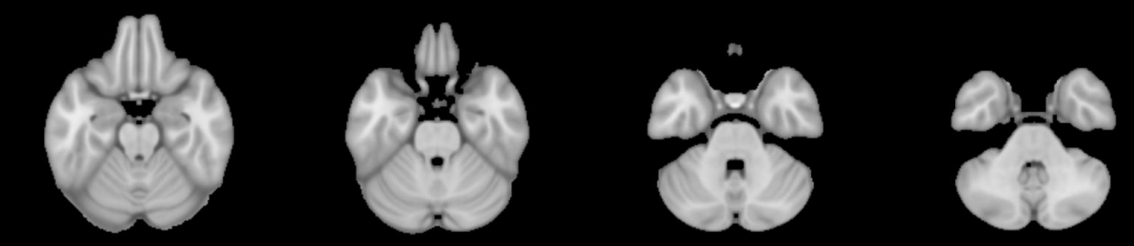

$z=-22$

\section{$2.3 \quad Z \quad 4.47$}

Figure 15 - The regions of activation for the ToM task contrasting the visualization of the physical causality cartoon stories and the affective ToM ones. 


\section{DISCUSSION}

This study involved the cooperation of five children with ADHD successfully performing two tasks to explore the neural substrates associated with social cognition and emotion recognition. The first task, Facial Emotion Perception Task (FEPT), demonstrated that the children had a harder time identifying emotions other than happiness, but in particular, anger. This task also showed regions of activation in the prefrontal cortex and insula, as expected. The second task, Theory of Mind (ToM), showed that the participants were able to (on average) correctly predict the ending to over $74 \%$ of the cartoon stories (i.e., physical causality accuracy $=74 \%$ and affective ToM accuracy $=78 \%$ ). However, the affective ToM stories had two more questions answered correctly. The activation maps from this task also showed similar regions of activation (i.e., the prefrontal cortex and insula), with the addition of the amygdala (left side; aToM > Rest (-20, $-9,-14)$ and PC $>$ Rest $(-20,-5,-25))$.

\section{A. Facial Emotion Perception Task}

In the first task, Facial Emotion Perception Task, the participants were able to accurately categorize $75 \%$ of the emotions and had an average response time of $1122.03 \mathrm{~ms}$ for the emotional expressions and $25.0 \mathrm{~ms}$ for the animal types. These finding confirm the hypothesis that the participants would have slower response times for the emotions than the animals (Hypothesis 2). The second part of Hypothesis 2 regarded the emotion happiness as being the easiest to correctly categorize of the four emotions. This as well was confirmed as "happy" 
had the fewest incorrect responses at six of 150. The other three emotions (fear, angry, and neutral) each averaged about ten incorrect. Similar results (i.e., "happy" having the best accuracy and "angry" having one of the worst accuracies) were also seen in a study by Rapport et al. in 2002 where adult participants with ADHD and healthy controls were told to identify black and white emotional and animal images. When analyzing the response times for each emotion, it appeared that the neutral and fearful facial expressions took longer to respond to, when compared to the happy and angry emotions. These findings were validated when looking at the results of the t-test on the emotional pairs in Table 2. There we see that the differences between the means for the response times of the neutral-fearful and angry-happy pairs did not differ significantly $(P>0.05)$.

From the activation maps, it can be seen that a large portion of the anterior and posterior aspects of the brain were activated during the Facial Emotion Perception Task while the participants viewed facial expressions and the animal types. When analyzing the contrast of faces $>$ animals (Figure 10), two regions of particular interest emerged: the posterior cingulate and the parahippocampal gyrus. The posterior cingulate is associated with memory, emotional salience, and the mediation of interactions between emotion and memory (Maddock et al., 2001 and Maddock et al., 2003). The parahippocampus is known to be associated with memory encoding and retrieval (Tulving et al., 1996). Therefore, the activation of these two regions during face viewing compared to animals may be attributed to significant involvement in affective processing and memory. 
When looking at the activation maps rendered from the participants viewing and discriminating the faces and the animals, it should be recognized that both activation maps yielded mostly left lateralized anterior brain regions. These regions appeared to be similar to some of the intrinsic connectivity networks (ICNs) introduced by Laird et al. in 2011. Although some of our regions display left-lateralized tendencies, the ICNs that our data most closely resemble relate to networks associated with emotional and autonomic processes, motor and visuospatial integration, coordination, and execution, visual perception, and language interpretation (i.e., semantic, phonologic, and orthographic language skills). Two of the larger and more distinguishable resemblances to ICNs dealt with visuospatial processing and reasoning, executive function, and affective and interceptive processing. The regions that most notably represent these areas are the prefrontal cortex (i.e., dorsolateral prefrontal cortex - dIPFC and ventromedial prefrontal cortex - vmPFC; BA 9/10/12/13/32/46) and insula (BA 13). These regions along with the amygdala were hypothesized to be activated during the FEPT task due to their involvement with emotion regulation and response. Surprisingly, the amygdala was not activated during the viewing of the emotional faces or the animals. When searching the scientific literature, findings concerning the amygdala and emotion perception tasks in ADHD patients have demonstrated mixed results. In a 2008 study by Herpertz et al., thirteen male adolescents with ADHD and thirteen typically developing controls were shown visually arousing negative (e.g., wounded children, scenes of violence), positive (e.g., exciting sports scenes, happy people), and neutral images (e.g., 
agriculture, surroundings) in order to gather new insight on emotional processing. The findings indicated viewing the negative versus neutral pictures caused increased regions of activation in two left hemisphere clusters (insula, putamen, and pallidum, and thalamus and caudate nucleus) in healthy male adolescents than in male adolescents with ADHD. When comparing these same groups of males, the left inferior temporal gyrus was observed to demonstrate increased activation for healthy controls when viewing positive vs. neutral pictures. No regions were found with higher activations when contrasting the males with ADHD and the healthy controls in both the negative versus neutral and positive versus neutral contrast. A region of interest $(\mathrm{ROI})$ analysis was also conducted to target particular brain regions (e.g., amygdala and prefrontal regions such as the anterior cingulate cortex, orbitofrontal and medial frontal gyri) and focus on activations in those regions as they are associated with emotional regulation. The results showed that there was greater activation in the left insula of the healthy males, and no activations in the amygdala, anterior cingulate cortex, or prefrontal areas. This study demonstrated that there was no indication of abnormal functioning associated with the amygdala or prefrontal regions in adolescent males with ADHD. In contrast, a study by Posner et al. in 2011 yielded results indicating that the right amygdala had greater activation in the fifteen ADHD adolescent participants than in the fifteen healthy controls during a subliminal presentation of fearful faces. The amygdala was also found to have greater connectivity with the lateral prefrontal cortex (LPFC) during the task. These mixed results may be the result of small sample sizes (Herpertz et al. - thirteen 
and Posner et al. - fifteen), the gender differences between the groups (Herpertz et al. - thirteen males versus Posner et al. - thirteen males and two females), and possibly even ethnic differences (Herpertz et al. - unknown and Posner et al. - White/Caucasian). The study additionally looked at the effects of prescribed stimulants and discovered that the stimulants suppressed the increased activation and subsequently normalized the level of activity in the right amygdala and the connectivity between the amygdala and LPFC. Both of the above mentioned studies illustrate amygdala activations, which is contrary to our findings, but does provide insight to an abnormal activation that is occurring in the amygdala. More recently, Hulvershorn et al. has observed abnormal amygdala functional connectivity with emotional lability (i.e., rapid changes in mood due to the occurrence of strong emotions or feelings) in children with ADHD while looking at intrinsic functional connectivity (i.e., resting state) (2014).

\section{B. Theory of Mind Task}

The Theory of Mind task yielded similar accuracies for the physical causality and the affective Theory of Mind story cartoons (i.e., $74 \%$ and $78 \%$ respectively). The descriptive statistics in Table 3 show that although the mean accuracies were similar, the mean response times were statistically different (i.e., $3099.4 \mathrm{~ms}$ and $3799.8 \mathrm{~ms} ; \mathrm{P}<0.05)$. The first finding regarding the accuracies, invalidates Hypothesis 3, which predicted that the affective Theory of Mind questions would take longer to answer and would have more incorrect answers. The former part was confirmed, but the latter part was not. This makes us think that either the 
children with $A D H D$ are better at picking endings to stories that require empathy over those that are cause and effect, and therefore indicating that these children may in fact not be showing signs of a lack of empathy when compared to typically developing children, or our small sample size or low question count was holding back our results.

When looking at the activation maps, it is apparent that some of the same regions that were activated during the Facial Emotion Perception Task were also activated during the Theory of Mind task. The insula (right in aToM and PC, but bilaterally in aToM>PC; BA 13) and prefrontal cortex (dIPFC and vmPFC; BA $9 / 12 / 13 / 32 / 44 / 46$ ) were among the most significant regions activated during this task. Unlike the FEPT task, there were small activations in the amygdala (left side; PC: $(-20,-5,-25)$ and aToM: $(-20,-9,-14))$. It is particularly interesting that the amygdala did not appear to be activated during the Facial Emotion Perception Task, but it was for the Theory of Mind task, especially given that the amygdala has been seen to be involved with emotion perception, decisionmaking, controlling aggression, and storing memories (Morris et al., 1998; Gupta et al., 2011; Brink, 2008; Hamann, 2005). With this amygdala activation, the portion of Hypothesis 1 that deals with the Theory of Mind task was confirmed (i.e., the prefrontal cortex, insula, and amygdala were activated). These regions appear to resemble many of the same ICN networks as in the FEPT task (Laird et al., 2011). There are three distinguishing differences between the resembled ICN for the two tasks. The first is that during the FEPT task only, there appeared to be neural activations that are similar to those associated with language 
interpretation networks. The other two differences are additional ICN resemblances for the ToM task. One of the new networks appears to be related to cognitive processing (i.e., reasoning, attention, inhibition, and memory), while the other appears to be the default mode. The default mode network as mentioned earlier has been known to be comprised of the ventromedial prefrontal cortex (vmPFC), posterior cingulate, and precuneus. With the exception of the PCC, it appears that the other regions were activated while performing the affective Theory of Mind and physical causality questions. Similar to the findings of Weissman's et al. (2006), our results could show a failure to fully suppress the DMN. The fact that the aToM cartoons took longer to answer but had two fewer question answered incorrectly, could support this hypothesis as they indicate that the participants were trying harder to answer the aToM cartoons.

\section{Methodological Considerations}

Some possible errors could be attributed to the excessive head motion during the tasks, in particularly during the Theory of Mind task. During the Theory of Mind task, the participants had a habit of shifting their head up when the choice endings slide introduced the three smaller story cartoons that were shifted up from the previous slide. Other issues of great concern include the low sample size and the lack of a typically developing control population. With the addition of

more participants, additional analyses could have been performed to possibly reveal further findings. For example, more data from FEPT potentially could have yielded separate emotional activation maps and more contrast maps. Different 
results could have arisen from statistical analyses with larger inputs. By having controls to compare the participants to, contrast analyses could have been done to show group differences in accuracy, response times, and neural activations.

\section{Future Work}

The research performed in this study has the potential to provide new insights into functional deficits associated with ADHD, particularly related to social cognition and emotion recognition. Through the use of the Facial Emotion Perception Task and Theory of Mind task, we were able to identify activated brain regions. These regions can then be compared to those of healthy developing children to determine the whole-brain networks impacted by ADHD. By also incorporating resting state data and behavioral information (i.e., reaction times and accuracy from the tasks), "sub-groupings" of ADHD could emerge, which can ultimately mean that clinical advances can be made for specific types of ADHD.

\section{E. Conclusion}

The findings from this study demonstrate the feasibility of using functional magnetic resonance imaging in young children with attention-deficit/hyperactivity disorder during tasks that elicit categorization of emotional expressions and correct story endings. The Facial Emotion Perception Task data confirmed one of our hypotheses by showing that the participants had an easier and quicker time identifying a "happy" emotion, and were slower to identify fearful, angry, and 
neutral expressions. Surprisingly for the Theory of Mind data, our hypothesis regarding the participants being able to more accurately and quickly predict the physical causality endings, was proved incorrect. These observations along with similar frontal and temporal neural activations findings (between the tasks and compared to literature) allude to similar underlying networks. These cognitive findings could be associated with failure to suppress the default mode network. Further experiments and analyses will be necessary to fully address this possible cognitive dysfunction or any other neural dysfunction there may be. 


\section{References}

American Psychiatric Association. (2013). Diagnostic and Statistical Manual of Mental Disorders (DSM-5®). American Psychiatric Pub.

Baron-Cohen, S., \& Ring, H. (1994). A model of the mindreading system: Neuropsychological and neurobiological perspectives. Origins of an understanding of mind, 183-207.

Baron-Cohen, S., Ring, H., Moriarty, J., Schmitz, B., Costa, D., \& Ell, P. (1994). The brain basis of theory of mind: the role of the orbito-frontal region. British Journal of Psychiatry, 165, 640-649.

Baron-Cohen, S., Ring, H. A., Wheelwright, S., Bullmore, E. T., Brammer, M. J., Simmons, A., \& Williams, S. C. (1999). Social intelligence in the normal and autistic brain: an fMRI study. European Journal of Neuroscience, 11(6), 18911898.

Brink, T. L. (2008). Psychology a student friendly approach. Unit, 5, 88.

Brothers, L. (1990). The social brain: a project for integrating primate behavior and neurophysiology in a new domain. Concepts in Neuroscience, 1, 27-51.

Brotman, M. A., Rich, B. A., Guyer, A. E., Lunsford, J. R., Horsey, S. E., Reising, M. M., ... \& Leibenluft, E. (2010). Amygdala activation during emotion processing of neutral faces in children with severe mood dysregulation versus ADHD or bipolar disorder. American Journal of Psychiatry.

Castellanos, F. X., Margulies, D. S., Kelly, C., Uddin, L. Q., Ghaffari, M., Kirsch, A., ... \& Sonuga-Barke, E. J. (2008). Cingulate-precuneus interactions: a new locus of dysfunction in adult attention-deficit/hyperactivity disorder. Biological psychiatry, 63(3), 332-337.

Colby, J. B., Rudie, J. D., Brown, J. A., Douglas, P. K., Cohen, M. S., \& Shehzad, Z. (2012). Insights into multimodal imaging classification of ADHD. Frontiers in systems neuroscience, 6 .

Collins, D. L., Neelin, P., Peters, T. M., \& Evans, A. C. (1994). Automatic 3D intersubject registration of MR volumetric data in standardized Talairach space. Journal of computer assisted tomography, 18(2), 192-205.

Cortese, S., Kelly, C., Chabernaud, C., Proal, E., Di Martino, A., Milham, M. P., \& Castellanos, F. X. (2012). Toward systems neuroscience of ADHD: a metaanalysis of $55 \mathrm{fMRI}$ studies. American Journal of Psychiatry. 
Costa Dias, T. G., Iyer, S. P., Carpenter, S. D., Cary, R. P., Wilson, V. B., Mitchel, S. H., ... \& Fair, D. A. (2015). Characterizing heterogeneity in children with and without ADHD based on reward system connectivity. Developmental cognitive neuroscience, 11, 155-174.

Cox, R. W. (1996). AFNl: software for analysis and visualization of functional magnetic resonance neuroimages. Computers and Biomedical research, 29(3), 162-173.

Ekman, P., \& Friesen, W. V. (1975). Pictures of facial affect. consulting psychologists press.

Gallagher, H. L., \& Frith, C. D. (2003). Functional imaging of 'theory of mind'. Trends in cognitive sciences, 7(2), 77-83.

Griffiths, D. J. (2005). Introduction to quantum mechanics. Pearson Education India.

Gupta, R., Koscik, T. R., Bechara, A., \& Tranel, D. (2011). The amygdala and decision-making. Neuropsychologia, 49(4), 760-766.

Hamann, S. (2005). Sex differences in the responses of the human amygdala. The Neuroscientist, 11(4), 288-293.

Herpertz, S. C., Huebner, T., Marx, I., Vloet, T. D., Fink, G. R., Stoecker, T., ... \& Herpertz-Dahlmann, B. (2008). Emotional processing in male adolescents with childhood-onset conduct disorder. Journal of Child Psychology and Psychiatry, 49(7), 781-791.

Huettel, S. A., Song, A. W., \& McCarthy, G. (2004). Functional magnetic resonance imaging (Vol. 1). Sunderland: Sinauer Associates.

Hulvershorn, L. A., Mennes, M., Castellanos, F. X., Di Martino, A., Milham, M. P., Hummer, T. A., \& Roy, A. K. (2014). Abnormal amygdala functional connectivity associated with emotional lability in children with attention-deficit/hyperactivity disorder. Journal of the American Academy of Child \& Adolescent Psychiatry, 53(3), 351-361.

Jenkinson, M., Beckmann, C. F., Behrens, T. E., Woolrich, M. W., \& Smith, S. M. (2012). Fsl. Neuroimage, 62(2), 782-790.

Laird, A. R., Fox, P. M., Eickhoff, S. B., Turner, J. A., Ray, K. L., McKay, D. R., ... \& Fox, P. T. (2011). Behavioral interpretations of intrinsic connectivity networks. Journal of cognitive neuroscience, 23(12), 4022-4037. 
Langenecker, S. A., Caveney, A. F., Giordani, B., Young, E. A., Nielson, K. A., Rapport, L. J., ... \& Kerber, K. (2007). The sensitivity and psychometric properties of a brief computer-based cognitive screening battery in a depression clinic. Psychiatry research, 152(2), 143-154.

Lauterbur, P. C. (1973). Image formation by induced local interactions: examples employing nuclear magnetic resonance.

Maddock, R. J., Garrett, A. S., \& Buonocore, M. H. (2001). Remembering familiar people: the posterior cingulate cortex and autobiographical memory retrieval. Neuroscience, 104(3), 667-676.

Maddock, R. J., Garrett, A. S., \& Buonocore, M. H. (2003). Posterior cingulate cortex activation by emotional words: fMRI evidence from a valence decision task. Human brain mapping, 18(1), 30-41.

Mitchell, R. L., \& Phillips, L. H. (2015). The overlapping relationship between emotion perception and theory of mind. Neuropsychologia, 70, 1-10.

Morris, J. S., Friston, K. J., Büchel, C., Frith, C. D., Young, A. W., Calder, A. J., \& Dolan, R. J. (1998). A neuromodulatory role for the human amygdala in processing emotional facial expressions. Brain, 121(1), 47-57.

Pelham Jr, W. E., \& Hoza, B. (1996). Intensive treatment: A summer treatment program for children with ADHD.

Premack, D., \& Woodruff, G. (1978). Does the chimpanzee have a theory of mind?. Behavioral and brain sciences, 1(04), 515-526.

Poldrack, R. A., Mumford, J. A., \& Nichols, T. E. (2011). Handbook of functional MRI data analysis. Cambridge University Press.

Posner, J., Nagel, B. J., Maia, T. V., Mechling, A., Oh, M., Wang, Z., \& Peterson, B. S. (2011). Abnormal amygdalar activation and connectivity in adolescents with attention-deficit/hyperactivity disorder. Journal of the American Academy of Child \& Adolescent Psychiatry, 50(8), 828-837.

Raichle, M. E., MacLeod, A. M., Snyder, A. Z., Powers, W. J., Gusnard, D. A., \& Shulman, G. L. (2001). A default mode of brain function. Proceedings of the National Academy of Sciences, 98(2), 676-682.

Rapport, L. J., Friedman, S. L., Tzelepis, A., \& Van Voorhis, A. (2002). Experienced emotion and affect recognition in adult attention-deficit hyperactivity disorder. Neuropsychology, 16(1), 102. 
Smith, S. M., Jenkinson, M., Woolrich, M. W., Beckmann, C. F., Behrens, T. E., Johansen-Berg, H., ... \& Niazy, R. K. (2004). Advances in functional and structural MR image analysis and implementation as FSL. Neuroimage, 23, S208-S219.

Sebastian, C. L., Fontaine, N. M., Bird, G., Blakemore, S. J., De Brito, S. A., McCrory, E. J., \& Viding, E. (2012). Neural processing associated with cognitive and affective Theory of Mind in adolescents and adults. Social cognitive and affective neuroscience, 7(1), 53-63.

Talairach, J., \& Tournoux, P. (1988). Co-planar stereotaxic atlas of the human brain: 3-Dimensional proportional system: An approach to cerebral imaging. Stuttgart: Georg Thieme.

Tulving, E., Markowitsch, H. J., Craik, F. I., Habib, R., \& Houle, S. (1996). Novelty and familiarity activations in PET studies of memory encoding and retrieval. Cerebral Cortex, 6(1), 71-79.

Uddin, L. Q., Kelly, A. C., Biswal, B. B., Margulies, D. S., Shehzad, Z., Shaw, D., ... \& Milham, M. P. (2008). Network homogeneity reveals decreased integrity of default-mode network in ADHD. Journal of neuroscience methods, 169(1), 249254.

Uekermann, J., Kraemer, M., Abdel-Hamid, M., Schimmelmann, B. G., Hebebrand, J., Daum, I., ... \& Kis, B. (2010). Social cognition in attention-deficit hyperactivity disorder (ADHD). Neuroscience \& Biobehavioral Reviews, 34(5), 734-743.

Vuilleumier, P., Armony, J. L., Driver, J., \& Dolan, R. J. (2001). Effects of attention and emotion on face processing in the human brain: an event-related fMRI study. Neuron, 30(3), 829-841.

Weisenbach, S. L., Rapport, L. J., Briceno, E. M., Haase, B. D., Vederman, A. C., Bieliauskas, L. A., ... \& Langenecker, S. A. (2012). Reduced emotion processing efficiency in healthy males relative to females. Social cognitive and affective neuroscience, nss137.

Weissman, D. H., Roberts, K. C., Visscher, K. M., \& Woldorff, M. G. (2006). The neural bases of momentary lapses in attention. Nature neuroscience, 9(7), 971978.

Woolrich, M. W., Jbabdi, S., Patenaude, B., Chappell, M., Makni, S., Behrens, T., ... \& Smith, S. M. (2009). Bayesian analysis of neuroimaging data in FSL. Neuroimage, 45(1), S173-S186. 